\title{
Robust semi-device-independent certification of all pure bipartite maximally entangled states via quantum steering
}

\author{
Harshank Shrotriya, ${ }^{1}$ Kishor Bharti, ${ }^{1}$ and Leong-Chuan Kwek $\oplus^{1,2,3}$ \\ ${ }^{1}$ Centre for Quantum Technologies, National University of Singapore, Singapore 117543, Singapore \\ ${ }^{2}$ MajuLab, CNRS-UNS-NUS-NTU International Joint Research Unit, Singapore UMI 3654, Singapore \\ ${ }^{3}$ National Institute of Education, Nanyang Technological University, Singapore 637616, Singapore
}

(Received 12 August 2020; revised 17 March 2021; accepted 7 July 2021; published 27 July 2021)

\begin{abstract}
The idea of self-testing is to render guarantees concerning the inner workings of a device based on the measurement statistics. It is one of the most formidable quantum certification and benchmarking schemes. Recently it was shown [A. Coladangelo, K. T. Goh, and V. Scarani, Nat. Commun. 8, 15485 (2017)] that all pure bipartite entangled states can be self-tested in the device-independent scenario by employing subspace methods introduced by Yang and Navascués [Phys. Rev. A 87, 050102(R) (2013)]. Here, we have adapted their method to show that any bipartite pure entangled state can be certified in the semi-device-independent scenario through quantum steering. Analogous to the tilted Clauser-Horne-Shimony-Holt inequality, we use a steering inequality called the tilted steering inequality for certifying any pure two-qubit entangled state. Furthermore, we use this inequality to certify any bipartite pure entangled state by certifying two-dimensional subspaces of the qudit state by observing the structure of the set of assemblages obtained on the trusted side after measurements are made on the untrusted side. As a feature of quantum state certification via steering, we use the notion of assemblage-based robust state certification to provide robustness bounds for the certification result in the case of pure maximally entangled states of any local dimension.
\end{abstract}

DOI: 10.1103/PhysRevResearch.3.033093

\section{INTRODUCTION}

Quantum certification and benchmarking become tasks of paramount importance as we advance towards the second quantum revolution [1]. One of the prominent approaches to device certification is self-testing [2-4]. The idea of selftesting is to provide guarantees regarding the inner working of a device based on the measurement statistics. Self-testing is an important task from the point of view of both quantum foundations research [5-8] as well as many quantum information processing tasks such as randomness generation [9-11], quantum cryptography $[3,12,13]$, and entanglement certification $[14,15]$. Self-testing as a method for certification of quantum states and measurements originated in the device-independent (DI) scenario [2,3,12], taking advantage of Bell nonlocality [16] to make the claims for certification. Since then, many important results have been achieved for device-independent self-testing such as self-testing of all pure bipartite entangled states [17], self-testing of all multipartite entangled states that admit a Schmidt decomposition [18], and sequential [19] and parallel self-testing of many Einstein-PodolskyRosen (EPR) pairs [20,21] and Greenberger-Horne-Zeilinger states [22]. Apart from the DI scenario, other scenarios have

Published by the American Physical Society under the terms of the Creative Commons Attribution 4.0 International license. Further distribution of this work must maintain attribution to the author(s) and the published article's title, journal citation, and DOI. been explored such as self-testing local quantum systems using noncontextuality inequalities [23,24], self-testing in the prepare and measure scenario [25], self-testing of quantum circuits [26] and semi-device-independent (SDI) state certification based on EPR steering [27-29]. In [28], the authors are mainly concerned with showing improvements in robust state certification in the SDI scenario compared to DI scenario, while in [27], the authors have shown one-sided device-independent state certification of any pure two-qubit entangled state. Since the "self-testing" terminology was originally defined for the DI scenario, we refrain from using it in this paper and instead refer to our approach as SDI state certification.

Quantum steering [30-32] as a phenomenon is logically different from entanglement and nonlocality, lying midway between them. Nonlocal states can be shown to be steerable and steerable states can be shown to be entangled; however, nonlocality is studied in the DI scenario while steering is studied in the SDI scenario. Applications of quantum steering have been investigated in quantum key distribution (QKD) [33] using the BBM92 protocol [34], where the authors showed that in the SDI scenario obtainable key rates are higher and the required detector efficiencies are lower compared to the fully DI case. Steering-based randomness certification has been studied in [35-37]. In [37], the authors show maximal randomness generation in the SDI setting from any pure entangled full-Schmidt-rank state using the maximal violation of steering inequalities. See [38] for a comprehensive review on quantum steering and [39] for a review based on semidefinite programming. 
Self-testing in the DI scenario entails obtaining specific extremal correlations while treating the measurement devices as black boxes. Such extremal correlations violate inequalities such as the Clauser-Horne-Shimony-Holt (CHSH) inequality [40] maximally. Although SDI is a weaker notion and requires more assumptions than the fully DI case, the advantages of state certification in the SDI scenario are manifold. The additional assumptions help in alleviating mathematical difficulties such as establishing a tensor product structure in the multipartite case, or certification of complex measurements as described in [28], where state certification using the maximal violation of a steering inequality in the SDI scenario was shown to be advantageous in a laboratory setting [41]. Furthermore, as we show in this paper, the steering-based approach renders robust state certification tractable by simplifying the mathematical analysis required. Such robustness bounds were missing in previous DI self-testing results [17], which makes the steering-based approach favorable for experimental implementations [42-45]. Very recently, the authors of $[46,47]$ obtained robust self-testing results using correlations for different subsets of maximally entangled states; however, the correlations-based approach is nonconstructive in nature while in the steering case we have shown explicit bounds.

The assumptions required in the SDI scenario are naturally justified, such as in the case of delegated quantum computing [48]. In [29], the authors discuss rigidity of quantum steering correlations via sequential steering games and show that the overhead is reduced in the steering case compared to that of CHSH game rigidity. The authors further highlight the application of steering rigidity in verifying delegated quantum computation.

Moved by the advantages of the SDI scenario, it is natural to enquire whether all pure bipartite entangled states can be certified in the SDI scenario via quantum steering. Progress in the aforementioned direction was made in $[28,29]$ where the authors used a linear steering inequality for certifying the maximally entangled Bell pair. One-sided device-independent certification of any two-qubit pure entangled state was shown in [27], where the authors used two steering inequalities, the fine-grained inequality (FGI) [49] and analog CHSH [50] inequalities, for self-testing. However, the maximal violation of the FGI is not uniquely achieved by a particular target state, and the authors used the analog CHSH inequality, along with maximal violation of FGI, to uniquely ascertain which state had been certified.

In this work we have used the tilted steering inequality, the maximal violation of which uniquely certifies any two-qubit pure entangled state. Furthermore, by adapting the subspace methods of [17] for the SDI scenario, we provide a state certification scheme for any pure bipartite entangled state via quantum steering. Finally, we provide $O(\sqrt{\epsilon})$ robustness bounds for our steering-based state certification result for maximally entangled pure qudit states which are not known in the device-independent scenario.

\section{STEERING SCENARIO}

We have considered an SDI scenario where Alice and Bob share an entangled qudit state, such that Alice performs black- box measurements on her side and Bob performs tomographic reconstruction to obtain the exact density matrix of the states on his side after Alice's measurements.

Quantum steering was first formalised in [51], where the authors defined steerable states as those which do not admit a local hidden state (LHS) model for any assemblage generated on Bob's side. The assemblage (unnormalized state) $\sigma_{a \mid x}$ generated on Bob's side corresponding to outcome $a$ and measurement $x$ on Alice's side is said to admit an LHS model if it can be represented as a mixture of hidden states $\rho_{\lambda}$ originating from a probability distribution $\mu(\lambda)$ :

$$
\sigma_{a \mid x}=\int d \lambda \mu(\lambda) p(a \mid x, \lambda) \rho_{\lambda} .
$$

In accordance with quantum mechanics, the assemblage generated by performing projective measurements $M_{a \mid x}$ on Alice's side can be written as

$$
\sigma_{a \mid x}=\operatorname{tr}_{A}\left[\left(M_{a \mid x} \otimes I\right) \rho^{A B}\right],
$$

where $\sum_{a} M_{a \mid x}=\mathbb{1}$ and $M_{a \mid x} \geqslant 0 \forall a, x$. In [50], the authors described steerable states by modeling nonsteerable correlations using a local hidden variable (LHV)-LHS model. Given a bipartite system comprising of qubits with spatially separated parties Alice and Bob, denote $O_{A}$ and $O_{B}$ as the set of observables in the Hilbert space of Alice and Bob, respectively. An element in $O_{A}$ will be denoted by $x$ (similarly $y$ for $O_{B}$ ) and the outcomes corresponding to $x$ will be denoted by $a \in L(x)$ [similarly $b \in L(y)]$, where $L(x)[(L(y)]$ denotes the set of outcomes for the observable $x(y)$. The joint state $\rho^{A B}$ is said to be steerable if and only if it does not admit an LHVLHS decomposition for all $a \in L(x), b \in L(y), x \in O_{A}$, and $y \in O_{B}$. An LHV-LHS decomposition is based on the idea that Alice's outcomes are determined by a local hidden variable $\lambda$ and Bob's outcomes are determined by local measurements on a quantum state $\rho_{\lambda}$,

$$
p\left(a, b \mid x, y ; \rho^{A B}\right)=\sum_{\lambda} \mathfrak{p}(\lambda) \mathfrak{p}(a \mid x, \lambda) p\left(b \mid y, \rho_{\lambda}\right) .
$$

In the SDI scenario, the LHV-LHS model can be used to establish local bounds for linear expressions giving rise to steering inequalities; violation of such inequalities implies steering (see Appendix A1). Steerable weight as a quantifier of steering was proposed in [52] and was further shown to be a convex steering monotone in [53]. Considering a onesided device-independent scenario where Alice and Bob share a joint quantum state $\rho^{A B}$, an arbitrary set of assemblages $\left\{\sigma_{a \mid x}\right\}_{a, x}$ (a set of assemblages obtained overall outcomes and observables) can be decomposed as

$$
\sigma_{a \mid x}=p_{s} \sigma_{a \mid x}^{S}+\left(1-p_{s}\right) \sigma_{a \mid x}^{U S} \quad \forall a, x,
$$

where $0 \leqslant p_{s} \leqslant 1, \sigma_{a \mid x}^{S}$ is a steerable assemblage, and $\sigma_{a \mid x}^{U S}$ is an unsteerable assemblage, i.e., it has an LHS decomposition. The weight of the steerable part $p_{s}$ minimized over all possible decompositions of $\left\{\sigma_{a \mid x}\right\}_{a, x}$ gives the steerable weight $\mathrm{SW}\left(\left\{\sigma_{a \mid x}\right\}_{a, x}\right)$ of that assemblage set.

\section{SDI STATE CERTIFICATION}

Self-testing was originally introduced in the DI scenario where the maximal violation of the $\mathrm{CHSH}$ inequality was used 
to self-test the Bell state $[3,12]$. Such self-testing procedures are based on obtaining extremal correlations $p(a, b \mid x, y)=$ $\operatorname{tr}\left[\left(M_{a \mid x} \otimes N_{b \mid y}\right) \rho^{A B}\right]$, obtained by performing quantum measurements $M_{a \mid x}$ (acting on $\mathcal{H}_{A}$ ) and $N_{b \mid y}$ (acting on $\mathcal{H}_{B}$ ) on the joint quantum state $\rho^{A B} \in \mathcal{H}_{A} \otimes \mathcal{H}_{B}$, such that the correlations $p(a, b \mid x, y)$ achieve the quantum supremum of a Bell inequality and the quantum states that achieve the extremal correlations are unique up to local isometries. Formally defined, the extremal correlations $p(a, b \mid x, y)$ self-test the state and measurements $\left\{|\bar{\psi}\rangle, \bar{M}_{a \mid x}, \bar{N}_{b \mid y}\right\}$ if for all states and measurements $\left\{|\psi\rangle, M_{a \mid x}, N_{b \mid y}\right\}$ compatible with $p(a, b \mid x, y)$, there exists an isometry $\Phi=\Phi_{A} \otimes \Phi_{B}$ where $\Phi_{A}: \mathcal{H}_{A} \mapsto \mathcal{H}_{A} \otimes$ $\mathcal{H}_{A^{\prime}}$ and $\Phi_{B}: \mathcal{H}_{B} \mapsto \mathcal{H}_{B} \otimes \mathcal{H}_{B^{\prime}}$ such that

$$
\begin{aligned}
\Phi\left(|\psi\rangle_{A B}\right) & =\mid \text { junk }\rangle_{A B}|\bar{\psi}\rangle_{A^{\prime} B^{\prime}}, \\
\Phi\left(M_{a \mid x} \otimes N_{b \mid y}|\psi\rangle_{A B}\right) & =\mid \text { junk }\rangle_{A B}\left(\bar{M}_{a \mid x} \otimes \bar{N}_{b \mid y}|\bar{\psi}\rangle_{A^{\prime} B^{\prime}}\right),
\end{aligned}
$$

where $\bar{M}_{a \mid x}$ and $\bar{N}_{b \mid y}$ act on $\mathcal{H}_{A^{\prime}}$ and $\mathcal{H}_{B^{\prime}}$, respectively.

Coming to the SDI scenario, for certifying any pure bipartite entangled state, using Schmidt decomposition, our target state can be written as

$$
\left|\psi_{\text {target }}\right\rangle:=\sum_{i=0}^{d-1} c_{i}|i i\rangle
$$

where $0<c_{i}<1 \forall i$ and $\sum_{i=0}^{d-1} c_{i}^{2}=1$. In order to certify the target state (6), we intend to show the existence of an isometry $\Phi: \mathcal{H}_{A} \mapsto \mathcal{H}_{A} \otimes \mathcal{H}_{A^{\prime}}$ on Alice's side such that

$$
\begin{aligned}
\Phi\left(|\psi\rangle_{A B}\right) & =\mid \text { junk }\rangle_{A} \otimes\left|\psi_{\text {target }}\right\rangle_{A^{\prime} B}, \\
\Phi\left(M_{a \mid x}|\psi\rangle_{A B}\right) & =\mid \text { junk }\rangle_{A} \otimes \bar{M}_{a \mid x}\left|\psi_{\text {target }}\right\rangle_{A^{\prime} B},
\end{aligned}
$$

where $M_{a \mid x}$ acts on $\mathcal{H}_{A} ; \bar{M}_{a \mid x}$ acts on $\mathcal{H}_{A^{\prime}}$ and represents ideal measurements on Alice's side. Formally stated, this result is the following.

Result 1. In the SDI scenario, for any bipartite pure entangled state $\left|\psi_{\text {target }}\right\rangle$, there exists an ideal set of assemblages $\left\{\sigma_{a \mid x}\right\}_{a, x}^{\text {ideal }}$, where $x \in\{0,1,2\}$ and $a \in\{0, \ldots, d-1\}$, which when observed on Bob's side after Alice performs black-box measurements on their joint state $\rho^{A B}$ certifies the target state $\left|\psi_{\text {target }}\right\rangle$ and ideal measurements on Alice's side.

\section{PROOF SKETCH}

Here we provide a sketch of the proof for this result; the detailed proof is given in Appendix B and Fig. 1 depicts an overview of the SDI state certification scheme. Since our aim is to certify the state (6) by certifying the two-dimensional subspace-projected blocks, we first establish the tilted steering inequality (TSI) analogous to the tilted CHSH inequality [54], the maximal violation of which uniquely certifies any twoqubit pure entangled state $|\psi(\theta)\rangle=\cos \theta|00\rangle+\sin \theta|11\rangle$ in the SDI scenario. The TSI is a two-parameter inequality given by

$$
I_{\alpha, \beta} \equiv \alpha\left\langle A_{0}\right\rangle+\beta\left\langle A_{0} Z_{s}\right\rangle+\left\langle A_{1} X_{s}\right\rangle \leqslant \alpha+\sqrt{1+\beta^{2}},
$$

where $\beta, \alpha>0, A_{0}$ and $A_{1}$ refer to two-outcome black-box measurements on Alice's side, and $Z_{s}=\frac{|0\rangle\langle 0|-| 1\rangle\langle 1|}{2}$ and $X_{s}=$ $\frac{|0\rangle\langle 1|+| 1\rangle\langle 0|}{2}$ refer to local Schmidt basis measurements performed by Bob. In order to certify a given bipartite pure entangled state $|\psi(\theta)\rangle$, we require two additional constraints,

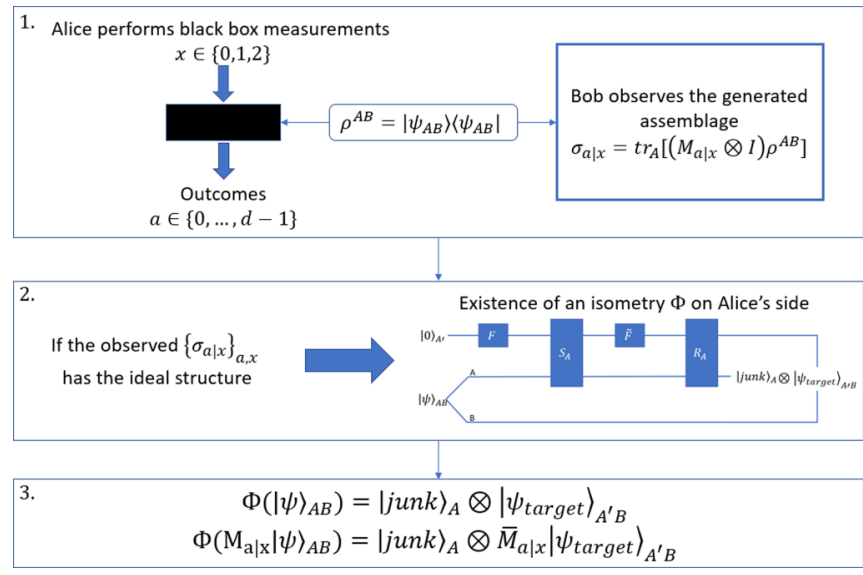

FIG. 1. SDI state certification scheme. Step 1: Alice performs uncharacterized measurements on her side with three measurement choices $x \in\{0,1,2\}$ and records the outcomes $a \in\{0, \ldots, d-1\}$. For each measurement $x$ and outcome $a$, Bob measures the assemblage generated on his side, $\sigma_{a \mid x}$. The experiment is repeated several times for each measurement setting $x$ to build the complete assemblage set on Bob's side. Step 2: If the observed assemblage set $\left\{\sigma_{a \mid x}\right\}_{a, x}$ has the ideal structure (see Appendix B1) then the isometry $\Phi$ (see Fig. 2) can be shown to exist on Alice's side. Step 3: The isometry $\Phi$ certifies the target state (6) and the ideal measurements as shown in Lemma 2 in Appendix B.

i.e., $\sin 2 \theta=1 / \beta$, and impose a condition on the parameters $\alpha$ and $\beta$ such that $\beta^{2}=\alpha^{2}+1$. Under these two conditions the state $|\psi(\theta)\rangle$ maximally violates TSI uniquely and this maximal violation is achieved when Alice's local Schmidt basis measurements are parallel to that of Bob's; i.e., Alice makes $A_{0}=\frac{|0\rangle\langle 0|-| 1\rangle\langle 1|}{2}$ and $A_{1}=\frac{|0\rangle\langle 1|+| 1\rangle\langle 0|}{2}$ measurements.

In order to calculate the local and quantum bounds for the linear expression $I_{\alpha, \beta}$, we use the concepts defined earlier, namely, the LHV-LHS model and steerable weight. In the LHV-LHS model, Alice's measurement outcomes are determined using a probabilistic LHV model and Bob's outcomes come from local measurements on a quantum state. We calculate the local bound for TSI within the LHV-LHS model by individually maximizing the terms of $I_{\alpha, \beta}$ over the allowed set of outcome probabilities for Alice and Bob. For calculating the quantum bound, we first consider a property of steerable weight $\left[\mathrm{SW}\left(\sigma_{a \mid x}\right)\right]$ given in [39]; $\mathrm{SW}\left(\sigma_{a \mid x}\right)$ is bounded by any convex function $f(\cdot)$ of that assemblage, where $f(\cdot)$ can be the violation of a steering inequality. Using this property, it can be further shown that

$$
\mathrm{SW}\left(\sigma_{a \mid x}\right) \geqslant \frac{f\left(\sigma_{a \mid x}\right)-f_{\max }^{\mathrm{LHS}}}{f_{\max }-f_{\max }^{\mathrm{LHS}}},
$$

where $f_{\max }$ and $f_{\max }^{\mathrm{LHS}}$ are the maximal value of $f(\cdot)$ among all possible assemblages and among all possible LHS assemblages, respectively. From the above equation it can be inferred that $\mathrm{SW}$ of an assemblage that gives the maximal violation of a steering inequality must be greater than or equal to 1 , which is in fact the maximum value that $\mathrm{SW}$ can take. Hence, SW of the assemblage that achieves the maximal violation must be 1 . Then we show that the quantum bound cannot be achieved by an assemblage generated from a 
mixed two-qubit entangled state using (9) and a lemma from [27]. Having eliminated mixed entangled states, we maximize the expression $\alpha\left\langle A_{0}\right\rangle+\beta\left\langle A_{0} B_{0}\right\rangle+\left\langle A_{1} B_{1}\right\rangle$ over all general projective measurements performed by Alice and Bob on a general pure two-qubit entangled state $|\psi(\theta)\rangle=\cos \theta|00\rangle+$ $\sin \theta|11\rangle$ where $0<\theta<\pi / 2$, thus giving the quantum bound $\sqrt{2\left(1+\alpha^{2}+\beta^{2}\right)}$. Kindly note that since we fix the local bases to be the Schmidt bases we cannot write the optimal Bob measurements as $Z$ and $X$. However, the local bases can always be rotated to give measurement directions as $Z$ and $X$.

We show explicitly in Appendix A2 that for all values of $\alpha, \beta>0$, the quantum bound is always greater than or equal to the local bound; however, for the purpose of certifying the state $|\psi(\theta)\rangle$ we impose an additional condition $\beta^{2}=\alpha^{2}+1$ on the parameters such that the state $|\psi(\theta)\rangle$ achieves the maximal violation $\sqrt{2\left(1+\alpha^{2}+\beta^{2}\right)}$ which is the quantum bound. The detailed proofs for obtaining the local and quantum bounds are given in Appendix A.

Second, we look for a laboratory fingerprint of the qudit state which can be used to show the existence of an isometry, as are the correlations in the DI case; naturally this is achieved by using assemblages in the SDI scenario where we impose a $2 \times 2$ outer product structure on the ideal assemblages generated on Bob's side for certain measurements of Alice (see Appendix B1 for details). For certifying a general state of the form (6), we show that Alice needs to perform three $d$-outcome measurements on her side; the structure imposed on the assemblage set $\left\{\sigma_{a \mid x}\right\}_{a, x}^{\text {ideal }}$, where $a \in\{0, \ldots, d-1\}$ and $x \in\{0,1,2\}$, is such that for measurement settings $x \in$ $\{0,1\}$ the pairs $c_{2 m}|2 m, 2 m\rangle+c_{2 m+1}|2 m+1,2 m+1\rangle$ and for measurement settings $x \in\{0,2\}$ the pairs $c_{2 m+1} \mid 2 m+$ $1,2 m+1\rangle+c_{2 m+2}|2 m+2,2 m+2\rangle$ for $m=\left\{0, \ldots, \frac{d}{2}-1\right\}$ are certified, respectively. The intuition behind certifying two different sets of pairs, similar to the DI case [17], is that the maximal violation of tilted steering inequality certifies the corresponding two-dimensional normalized projections $\left|\psi_{m}\right\rangle=\frac{c_{2 m}|2 m, 2 m\rangle+c_{2 m+1}|2 m+1,2 m+1\rangle}{\sqrt{c_{2 m}^{2}+c_{2 m+1}^{2}}}$ or $\left|\psi_{m}\right\rangle=$ $\frac{c_{2 m+1}|2 m+1,2 m+1\rangle+c_{2 m+2}|2 m+2,2 m+2\rangle}{\sqrt{c_{2 m+1}^{2}+c_{2 m+2}^{2}}}$ of the target state (6). That is, the maximal violation of a particular $I_{\alpha, \beta}$ certifies a state of the form $|\psi(\theta)\rangle$ satisfying $\sin 2 \theta=\frac{1}{\beta}$ which certifies the ratio $\tan \theta=\frac{c_{2 m+1}}{c_{2 m}}$ or $\tan \theta=\frac{c_{2 m+2}}{c_{2 m+1}}$ of the coefficients. Hence, we obtain $d / 2$ relations between the coefficients from the set corresponding to measurements $x \in\{0,1\}$ and another $d / 2$ from the set corresponding to $x \in\{0,2\}$, thus uniquely determining the $d$ coefficients of the target state (6).

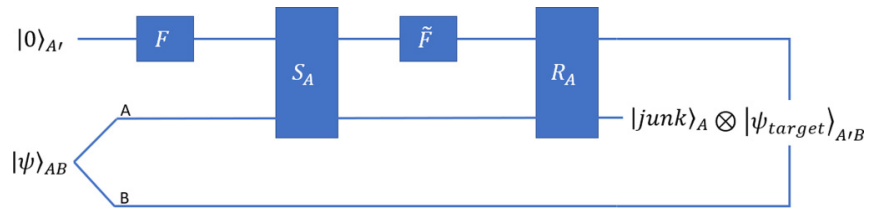

FIG. 2. Circuit diagram for the local isometry $\Phi$ on Alice's side. Gates $F$ and $\bar{F}$ are quantum Fourier transform and inverse quantum Fourier transform, respectively. Gate $R_{A A^{\prime}}$ is defined as $R_{A A^{\prime}}|\psi\rangle_{A B}|k\rangle_{A^{\prime}}=X_{A}^{(k)}|\psi\rangle_{A B}|k\rangle_{A^{\prime}}$, where $X_{A}^{(k)}$ are unitary operators and gate $S_{A A^{\prime}}$ is defined as $S_{A A^{\prime}}|\psi\rangle_{A B}|k\rangle_{A^{\prime}}=Z_{A}^{k}|\psi\rangle_{A B}|k\rangle_{A^{\prime}}$, where $Z_{A}:=\sum_{k=0}^{d-1} \omega^{k} P_{A}^{(k)}$ and $P_{A}^{(k)}$ are projections corresponding to measurement $x=0$ (refer to Appendix B for complete descriptions of all the gates)

Finally, to formally show the existence of an isometry (7), we construct projection operators $\left\{P_{A}^{(k)}\right\}_{k=0, \ldots, d-1}$ and unitaries $X_{A}^{(k)}$ on Alice's side which arise as a consequence of the structure imposed on the assemblage set $\left\{\sigma_{a \mid x}\right\}_{a, x}$. We further show that these projection operators and unitaries satisfy the condition

$$
X_{A}^{(k)} P_{A}^{(k)}|\psi\rangle=c_{k}|0, k\rangle \quad \forall k,
$$

which is sufficient for proving the existence of an isometry (Fig. 2) which certifies the target state and the ideal measurements (7) (see Lemma 2 in Appendix B for proof).

\section{ASSEMBLAGE-BASED ROBUST SDI STATE CERTIFICATION}

The notion of assemblage-based robust certification was introduced in [28], where the authors used their assemblagebased strategy to analytically derive better robustness bounds for certifying the singlet state, i.e., the maximally entangled two-qubit state, as compared to the bounds that were known for the device-independent case. To formalize this notion, the distance measure used is the trace distance between two quantum states, $\mathrm{D}\left(\rho_{1}, \rho_{2}\right)$, which is related to the Schatten- 1 norm $\left\|\rho_{1}-\rho_{2}\right\|_{1}$ as $\mathrm{D}\left(\rho_{1}, \rho_{2}\right)=\frac{1}{2}\left\|\rho_{1}-\rho_{2}\right\|_{1}$. Having established a notion of closeness of two quantum states, we state our robust state certification result as follows.

Main result. For the maximally entangled pure qudit state $\left|\bar{\psi}_{\text {target }}^{\max }\right\rangle=\frac{1}{\sqrt{d}} \sum_{i=0}^{d-1}|i i\rangle$ of dimension $d$ with $\bar{\rho}=$ $\left|\bar{\psi}_{\text {target }}^{\max }\right\rangle\left\langle\bar{\psi}_{\text {target }}^{\max }\right|$, if the experimentally obtained assemblages $\left\{\sigma_{a \mid x}\right\}_{a, x}$ and reduced state $\rho_{B}$ on Bob's side are close to the reference set of assemblages $\left\{\bar{\sigma}_{a \mid x}\right\}_{a, x}$ and reduced state $\bar{\rho}_{B}$ such that $\left\|\sigma_{a \mid x}-\bar{\sigma}_{a \mid x}\right\|_{1} \leqslant \epsilon \quad \forall a, x$ and $\mathrm{D}\left(\rho_{B}, \bar{\rho}_{B}\right) \leqslant \epsilon$, then

$$
\begin{gathered}
\mathrm{D}\left(|\Phi\rangle\langle\Phi|, \rho_{\text {junk }} \otimes \bar{\rho}_{A^{\prime} B}\right) \leqslant \frac{1}{2}\left(4 d^{2} \sqrt{\epsilon}+d^{3} \epsilon \sqrt{\epsilon}+d \epsilon\right), \\
\|\left|\Phi, M_{a \mid x}\right\rangle\left\langle\Phi, M_{a \mid x}\left|-\rho_{\text {junk }} \otimes\left(\bar{M}_{a \mid x} \otimes I_{B}\right)\right| \bar{\psi}_{\text {target }}^{\max }\right\rangle \bar{\psi}_{\text {target }}^{\max } \mid\left(\bar{M}_{a \mid x} \otimes I_{B}\right) \|_{1} \leqslant 4\left(d^{2}+d\right) \sqrt{\epsilon}+\epsilon,
\end{gathered}
$$

where $\quad|\Phi\rangle:=\Phi|\psi\rangle_{A B}|0\rangle_{A^{\prime}}, \quad\left|\Phi, M_{a \mid x}\right\rangle:=\Phi\left(M_{a \mid x} \otimes\right.$ $\left.I_{B}\right)|\psi\rangle_{A B}|0\rangle_{A^{\prime}}$, and $|\psi\rangle_{A B}$ is the purification of the reduced state $\rho_{B}$. (See Appendix $\mathrm{C}$ for detailed derivation.)

\section{DISCUSSION AND OPEN PROBLEMS}

In this paper, we have shown SDI certification of any pure bipartite entangled state by certifying two-dimensional subspace projections of the qudit state using a tilted steering 
inequality adapting the subspace methods previously employed in [6,17]. More importantly we derived robustness bounds for this certification in the case of the maximally entangled pure qudit state of dimension $d$; such explicit robust bounds are not known in the device-independent scenario. As shown in Appendix B2, in the ideal case Alice needs to make three measurements on her side in order to obtain the set of ideal assemblages which is sufficient for the certification task. Also, it can be easily seen that in the SDI scenario the same set of ideal measurements can be used to certify any qudit state of a given dimension. However, the ideal set of assemblages that we have used to show the existence of an isometry is not necessarily the only set of assemblages that certifies a given bipartite pure entangled state. It has been shown in [52] that, for a set of assemblages generated by performing two $d$-outcome projective measurements on any pure bipartite entangled state, a single linear steering inequality can be obtained which is maximally violated by that set of assemblages. Results in this direction could be used to show certification of a bipartite pure entangled state using a single steering inequality obtained using semidefinite programming based methods; furthermore, such a procedure could be shown to require only two measurements on Alice's side.

\section{ACKNOWLEDGMENTS}

The authors thank Debarshi Das for providing an improved and complete proof for Result 2 given in Appendix A3, which has been incorporated in the paper. Additionally, the authors would like to thank the National Research Foundation, Singapore and the Ministry of Education, Singapore, for financial support.

\section{APPENDIX A: TILTED STEERING INEQUALITY}

In this section we will show detailed calculations for obtaining the local and quantum bounds for the tilted steering expression $I_{\alpha, \beta} \equiv \alpha\left\langle A_{0}\right\rangle+\beta\left\langle A_{0} Z\right\rangle+\left\langle A_{1} X\right\rangle$. For obtaining the local bound we will maximize $I_{\alpha, \beta}$ for the correlations obeying the LHV-LHS model, and for obtaining the quantum bound (under the condition $\beta>\alpha>0$ ) we will consider arguments involving steerable weight and entangled states. Furthermore, we will show that the maximal violation of TSI uniquely certifies any two-qubit pure entangled state.

\section{Local bound of tilted steering inequality}

Let us first recap the LHV-LHS model here for completeness. Given a bipartite system comprising of qubits with spatially separated parties Alice and Bob, we denote $O_{A}$ and $O_{B}$ as the set of observables in the Hilbert space of Alice and Bob, respectively. An element in $O_{A}$ will be denoted by $x$ (similarly $y$ for $O_{B}$ ) and the outcomes corresponding to $x$ will be denoted by $a \in L(x)$ [similarly $b \in L(y)$ ], where $L(x)$ $[L(y)]$ denotes the set of outcomes for the observable $x(y)$. The joint state $\rho^{A B}$ is said to be steerable if and only if it does not admit a LHV-LHS decomposition for all $a \in L(x)$, $b \in L(y), x \in O_{A}$, and $y \in O_{B}$. A LHV-LHS decomposition is based on the idea that Alice's outcomes are determined by a local hidden variable $\lambda$ and Bob's outcomes are determined by local measurements on a quantum state $\rho_{\lambda}$,

$$
P\left(a, b \mid x, y ; \rho^{A B}\right)=\sum_{\lambda} \mathfrak{p}(\lambda) \mathfrak{p}(a \mid x, \lambda) p\left(b \mid y, \rho_{\lambda}\right) .
$$

For a given scenario (two measurements and two outcomes in this case) the correlations that have a LHV-LHS model form a convex set [41]; hence we can express any LHV-LHS model as a convex combination of extremal points of that convex set. This implies that we can decompose $\mathfrak{p}(a \mid x, \lambda) p\left(b \mid y, \rho_{\lambda}\right)$ into $\left.\sum_{\chi} \int d \xi \mathfrak{p}(\chi, \xi \mid \lambda) \delta_{a, f(A, \chi)} P\left(b|y ;| \psi_{\xi}\right\rangle\left\langle\psi_{\xi}\right|\right)$, where $\chi$ are the variables which determine the extremal outcome strategies for Alice via the function $f(A, \chi)$, and $\xi$ determines a pure state $\left|\psi_{\xi}\right\rangle$ for Bob. Thus we can simplify (A1) as

$$
P(a, b \mid x, y)=\sum_{\chi, \xi} \mathfrak{p}(\chi, \xi) \delta_{a, f(x, \chi)}\left\langle\psi_{\xi}\left|\Pi_{b \mid y}\right| \psi_{\xi}\right\rangle .
$$

To calculate the local bound of our tilted steering inequality we use the LHV-LHS model (A1) where Alice and Bob each have a choice between two dichotomic observables given by $\{x=0, x=1\},\{y=0, y=1\}$. As shown in Eq. (A2) we can express any joint probability distribution obeying the LHVLHS model as a convex sum of extremal points in that set. We will briefly review the method to obtain these extremal points for Alice's and Bob's sides as illustrated in [50]. For Alice's side with two observables $x=0, x=1$ with outcomes \pm 1 , the four deterministic strategies are given by

$$
\begin{aligned}
& p^{1 \mid 0}=1, p^{1 \mid 1}=1, \quad p^{1 \mid 0}=1, p^{1 \mid 1}=0, \\
& p^{1 \mid 0}=0, p^{1 \mid 1}=1, \quad p^{1 \mid 0}=0, p^{1 \mid 1}=0,
\end{aligned}
$$

and we attach these four strategies with four labels $\chi \in$ $\{1,2,3,4\}$. Moving ahead to Bob's side, since Bob's probabilities arise from measurements on a quantum state, they have to obey constraints such as uncertainty relations. Labeling the basis of eigenstates Bob's observable $y=0$ as $\{|1\rangle,|-1\rangle\}$, we can write the generalized projector for outcome 1 of observable $y=1$, parametrized by $\mu$ and $\phi$, as

$$
\begin{aligned}
\Pi_{1 \mid 1}= & \left(\sqrt{\mu}|1\rangle+\sqrt{1-\mu} e^{i \phi}|-1\rangle\right) \\
& \times\left(\sqrt{\mu}\langle 1|+\sqrt{1-\mu} e^{-i \phi}\langle-1|\right) .
\end{aligned}
$$

Similarly a general pure state can be written as

$$
\left|\psi_{\mu^{\prime}, \phi^{\prime}}\right\rangle=\sqrt{\mu^{\prime}}|1\rangle+\sqrt{1-\mu^{\prime}} e^{i \phi^{\prime}}|-1\rangle ;
$$

then the measurement probabilities of outcome 1 for $y=0$ and $y=1$ are

$$
\begin{aligned}
p^{1 \mid 0}\left(\mu^{\prime}, \phi^{\prime}\right) \equiv & \left\langle\psi_{\mu^{\prime}, \phi^{\prime}}\left|\Pi_{1 \mid 0}\right| \psi_{\mu^{\prime}, \phi^{\prime}}\right\rangle=\mu^{\prime}, \\
p^{1 \mid 1}\left(\mu^{\prime}, \phi^{\prime}\right)= & \mu \mu^{\prime}+(1-\mu)\left(1-\mu^{\prime}\right) \\
& +2 \sqrt{\mu(1-\mu) \mu^{\prime}\left(1-\mu^{\prime}\right)} \cos \left(\phi^{\prime}-\phi\right) .
\end{aligned}
$$

From the above equations, we see that the set of allowed quantum probabilities $\left(p^{1 \mid 0}, p^{1 \mid 1}\right)$ form the convex hull of an ellipse and the boundaries (extreme values) are achieved when $\cos \left(\phi^{\prime}-\phi\right)= \pm 1$. For the extreme points, we can reparametrize the ellipse as

$$
\begin{aligned}
& p^{1 \mid 0}(\xi)-\frac{1}{2}=\frac{1}{2}[\sqrt{\mu} \cos (\xi)-\sqrt{1-\mu} \sin (\xi)], \\
& p^{1 \mid 1}(\xi)-\frac{1}{2}=\frac{1}{2}[\sqrt{\mu} \cos (\xi)+\sqrt{1-\mu} \sin (\xi)] .
\end{aligned}
$$


Assuming the LHV-LHS model (A2) for probability distributions, the correlation terms of our inequality have a LHV-LHS model (LHV model for $\left\langle A_{0}\right\rangle$ ) if and only if they can be written as

$$
\begin{gathered}
\left\langle A_{0}\right\rangle=p^{1 \mid 0}-p^{-1 \mid 0}=2 p^{1 \mid 0}-1 \\
=\sum_{\chi} \mathfrak{p}(\chi)\left(2 p^{1 \mid 0}(\chi)-1\right), \\
\left\langle A_{0} B_{0}\right\rangle=P(a=b \mid x=0, y=0)-P(a=-b \mid x=0, y=0) \\
=\sum_{\chi} \int_{\xi} \mathfrak{p}(\chi, \xi)\left(2 p^{1 \mid 0}(\chi)-1\right)\left(2 p^{1 \mid 0}(\xi)-1\right), \quad(\mathrm{A} 9) \\
\left\langle A_{1} B_{1}\right\rangle=\sum_{\chi} \int_{\xi} \mathfrak{p}(\chi, \xi)\left(2 p^{1 \mid 1}(\chi)-1\right)\left(2 p^{1 \mid 1}(\xi)-1\right) .(\mathrm{A} 10)
\end{gathered}
$$

In the above expressions we have expressed the separate terms of our inequality as convex sums of extremal probabilities. From Eq. (A4) we see that $\mu=1 / 2$ refers to the case when the observables $y=0$ and $y=1$ correspond to orthogonal spin measurements, which is required in our case since Bob is making spin measurements along $Z, X$. In this case Eq. (A7) can further be simplified as [note that $\xi$ below is different from that used in Eq. (A7) but the symbol has been kept the same]

$$
\begin{aligned}
& 2 p^{1 \mid 0}(\xi)-1=\cos \xi, \\
& 2 p^{1 \mid 1}(\xi)-1=\sin \xi .
\end{aligned}
$$

Using Eqs. (A3) and (A11), we can further simplify the terms of our inequality for each value of $(\chi, \xi)$ as

$$
\begin{array}{lllll} 
& \chi=1 & \chi=2 & \chi=3 & \chi=4 \\
\left\langle A_{0}\right\rangle & 1 & 1 & -1 & -1 \\
\left\langle A_{0} B_{0}\right\rangle(\xi) & \cos \xi & \cos \xi & -\cos \xi & -\cos \xi \\
\left\langle A_{1} B_{1}\right\rangle(\xi) & \sin \xi & -\sin \xi & \sin \xi & -\sin \xi
\end{array}
$$

For the $\chi=1$ column in Eq. (A12), the following holds:

$$
\begin{aligned}
I_{\alpha, \beta} & \equiv \alpha\left\langle A_{0}\right\rangle+\beta\left\langle A_{0} B_{0}\right\rangle+\left\langle A_{1} B_{1}\right\rangle \\
& =\alpha+\beta \cos \xi+\sin \xi \\
& \leqslant \alpha+\sqrt{1+\beta^{2}} .
\end{aligned}
$$

One can similarly verify that for all columns in Eq. (A12), $I_{\alpha, \beta} \leqslant \alpha+\sqrt{1+\beta^{2}}$ is satisfied and therefore it must also be satisfied for any convex combination taken over $\chi$ and $\xi$ as shown in Eqs. (A8)-(A10). By obtaining this local bound, we can say that if for a quantum state $I_{\alpha, \beta}>\alpha+\sqrt{1+\beta^{2}}$ then the probability statistics obtained from that state do not admit a LHV-LHS decomposition and hence the state is steerable. As a result,

$$
I_{\alpha, \beta} \equiv \alpha\left\langle A_{0}\right\rangle+\beta\left\langle A_{0} Z\right\rangle+\left\langle A_{1} X\right\rangle \leqslant \alpha+\sqrt{1+\beta^{2}}
$$

is a valid steering inequality.

\section{Quantum bound of tilted steering inequality}

First let us look at the steerable weight of the set of assemblages that achieve the maximal violation of any general steering inequality. One of the interesting properties of steerable weight $\left[\mathrm{SW}\left(\sigma_{a \mid x}\right)\right]$ is that it is bounded by any convex function $f(\cdot)$ of that assemblage as given in [39], where $f(\cdot)$ can be the violation of a steering inequality. Using this property, it can be further shown that

$$
\operatorname{SW}\left(\sigma_{a \mid x}\right) \geqslant \frac{f\left(\sigma_{a \mid x}\right)-f_{\max }^{\mathrm{LHS}}}{f_{\max }-f_{\max }^{\mathrm{LHS}}},
$$

where $f_{\max }$ and $f_{\max }^{\text {LHS }}$ are the maximal values of $f(\cdot)$ among all possible assemblages and among all possible LHS assemblages, respectively. From the above equation it can be inferred that SW of an assemblage that gives the maximal violation of a steering inequality must be greater than or equal to 1 , which is in fact the maximum value that $\mathrm{SW}$ can take. Hence, SW of the assemblage that achieves the maximal violation must be 1 .

Now we will identify the candidate states which could achieve the maximal violation. Maximal violation of a steering inequality (maximally steerable states) implies that the initial state that generated the steerable assemblage must be a steerable state and since steerable states are a subset of entangled states, the maximally steerable state is an entangled state. It was proven in [27] that the steerable weight of an assemblage generated by an arbitrary bipartite qubit mixed entangled state cannot be equal to 1 . Therefore, in order to find the maximal quantum violation we focus only on pure two-qubit entangled states.

A general pure two-qubit entangled state can be written (using Schmidt decomposition) as

$$
|\psi(\theta)\rangle=\cos \theta|00\rangle+\sin \theta|11\rangle, \quad 0<\theta<\pi / 2 .
$$

Since we have taken the Schmidt decomposition as the generalized pure state, the expression that we are going to maximize would be

$$
\alpha\left\langle A_{0}\right\rangle+\beta\left\langle A_{0} B_{0}\right\rangle+\left\langle A_{1} B_{1}\right\rangle
$$

since Bob's optimal measurements might not be $Z$ and $X$ in the Schmidt basis; however, $B_{0}$ and $B_{1}$ projective measurement directions must remain perpendicular. This maximization of Eq. (A17) is equivalent to the maximization of the TSI since the local bases of Bob can always be rotated to make the Bob's optimal measurements as $Z$ and $X$; however, the form of our general entangled state $|\psi(\theta)\rangle$ would change in that case. We proceed to find the quantum bound by maximizing Eq. (A17) over all general pure twoqubit entangled states and general projective measurements on Alice's and Bob's sides which can be written as $A_{u}=$ $\vec{a}_{u} \cdot \vec{\sigma}$ and $B_{u}=\overrightarrow{b_{u}} \cdot \vec{\sigma}$, where $\vec{a}_{u}=\left(a_{u x}, a_{u y}, a_{u z}\right)$ and $\overrightarrow{b_{u}}=$ $\left(b_{u x}, b_{u y}, b_{u z}\right)$ are unit vectors with $\vec{b}_{0} \perp \vec{b}_{1}$. One can easily verify that the state $|00\rangle$ achieves the value $\alpha+\sqrt{1+\beta^{2}}$ with measurements $\vec{a}_{0}=\vec{a}_{1}=\hat{z}, \overrightarrow{b_{0}}=(\cos \mu, 0, \sin \mu)$ and $\vec{b}_{1}=(-\sin \mu, 0, \cos \mu)$ where $\cos \mu=1 / \sqrt{1+\beta^{2}}$; thus the quantum bound is always greater than or equal to the local bound for any choice of parameters $\alpha, \beta$. Let us now rewrite the state $|\psi(\theta)\rangle$ as $\rho=|\psi(\theta)\rangle\langle\psi(\theta)|$, where

$$
\rho=\frac{I}{4}+\cos 2 \theta \frac{\sigma_{z} \otimes I}{4}+\cos 2 \theta \frac{I \otimes \sigma_{z}}{4}+\sum_{i, j} T_{i j} \frac{\sigma_{i} \otimes \sigma_{j}}{4},
$$

and $T_{x x}=\sin 2 \theta, T_{y y}=-\sin 2 \theta, T_{z z}=1$, and $T_{i j}=0$ for $i \neq$ $j$. Consider the expression $I_{\beta} \equiv \beta\left\langle A_{0} B_{0}\right\rangle+\left\langle A_{1} B_{1}\right\rangle$, which is 
evaluated using $\rho$ and measurements $A_{u}$ and $B_{u}$ as

$$
I_{\beta}=\beta\left(\vec{a}_{0} \cdot T \overrightarrow{b_{0}}\right)+\vec{a}_{1} \cdot T \overrightarrow{b_{1}} .
$$

The expression above is maximized over all measurements $A_{0,1}$ and $B_{0,1}$ when $\vec{a}_{0} \| T \overrightarrow{b_{0}}$ and $\vec{a}_{1} \| T \overrightarrow{b_{1}}$, giving

$$
I_{\beta}=\beta\left|T \vec{b}_{0}\right|+\left|T \vec{b}_{1}\right|
$$

with $\overrightarrow{b_{0}}=(\cos \mu, 0, \sin \mu)$ and $\overrightarrow{b_{1}}=(-\sin \mu, 0, \cos \mu)$ since $\overrightarrow{b_{0}}$ and $\overrightarrow{b_{1}}$ can always be considered to be in the $X-Z$ plane. Thus it follows,

$$
I_{\beta}=\beta \sqrt{\sin ^{2} 2 \theta \cos ^{2} \mu+\sin ^{2} \mu}+\sqrt{\sin ^{2} 2 \theta \sin ^{2} \mu+\cos ^{2} \mu} .
$$

Differentiating the above expression with respect to $\mu$, we see maxima occur at $\cos ^{2} \mu=\frac{1-\beta^{2} \sin ^{2} 2 \theta}{\cos ^{2} 2 \theta\left(1+\beta^{2}\right)}$, giving $I_{\beta}^{\max }=$ $\sqrt{\left(1+\beta^{2}\right)\left(1+\sin ^{2} 2 \theta\right)}$. Moving on to the expression (A17), let us first make an observation about the single party correlation $\left\langle A_{0}\right\rangle$, i.e., for the state $\rho$ and general Pauli observable $A_{0}:-\cos 2 \theta \leqslant\left\langle A_{0}\right\rangle \leqslant \cos 2 \theta$ and the extremal values are achieved when $A_{0}= \pm \sigma_{z}$. Thus we have

$$
\begin{aligned}
& \alpha\left\langle A_{0}\right\rangle+\beta\left\langle A_{0} B_{0}\right\rangle+\left\langle A_{1} B_{1}\right\rangle \\
& \equiv \alpha\left\langle A_{0}\right\rangle+I_{\beta} \\
& \leqslant \alpha \cos 2 \theta+I_{\beta}^{\max } \\
& =\alpha \cos 2 \theta+\sqrt{\left(1+\beta^{2}\right)\left(1+\sin ^{2} 2 \theta\right) .}
\end{aligned}
$$

On maximizing the expression $\alpha \cos 2 \theta+$ $\sqrt{\left(1+\beta^{2}\right)\left(1+\sin ^{2} 2 \theta\right)}$ with respect to $\theta$ we see that maxima occur at $\sin ^{2} 2 \theta=\frac{1+\beta^{2}-\alpha^{2}}{1+\beta^{2}+\alpha^{2}}$ and the minima at $\sin 2 \theta=0$. Substituting the expression for $\sin 2 \theta$ corresponding to the maxima, we obtain the quantum bound of (A17) as $\sqrt{2\left(1+\beta^{2}+\alpha^{2}\right)}$. Note that the equality in the second line of Eq. (A22) exists only when certain conditions are fulfilled, which are

$$
\vec{a}_{0}\left\|T \overrightarrow{b_{0}}, \vec{a}_{1}\right\| T \overrightarrow{b_{1}} \text { and } A_{0}=\sigma_{z} .
$$

For these conditions to be true we require the $I_{\beta}^{\max }$ to occur at $\vec{b}_{1}=\hat{x}$ and $\overrightarrow{b_{0}}=\hat{z} \Rightarrow \cos \mu=0$, giving $\sin 2 \theta=\frac{1}{\beta}$. Since the maxima $\sqrt{2\left(1+\beta^{2}+\alpha^{2}\right)}$ occurs at $\sin ^{2} 2 \theta=\frac{1+\beta^{2}-\alpha^{2}}{1+\beta^{2}+\alpha^{2}}$, we have

$$
\frac{1+\beta^{2}-\alpha^{2}}{1+\beta^{2}+\alpha^{2}}=\frac{1}{\beta^{2}} \Rightarrow \beta^{2}=\alpha^{2}+1 .
$$

Thus for a general state $|\psi(\theta)\rangle$ [Eq. (A16)], if we design the TSI such that $\beta=1 / \sin 2 \theta$ and $\beta^{2}=\alpha^{2}+1$ then the quantum bound $\sqrt{2\left(1+\beta^{2}+\alpha^{2}\right)}(=2 \beta)$ is achieved only when Alice and Bob make the measurements $A_{0}=Z, A_{1}=X, B_{0}=$ $Z$, and $B_{1}=X$ in their respective local Schmidt bases.

\section{SDI certification of two-qubit pure entangled state using tilted steering inequality}

Lemma 1. In the steering scenario where Bob, the trusted party, performs mutually unbiased spin measurements along $Z$ and $X$, maximal violation $\left[\sqrt{2\left(1+\beta^{2}+\alpha^{2}\right)}\right]$ of the tilted steering inequality (with $\beta^{2}=\alpha^{2}+1$ ) occurs if and only if the two-qubit joint state shared between Alice and Bob is

$$
\begin{aligned}
|\psi(\theta)\rangle & =\cos \theta|00\rangle+\sin \theta|11\rangle, \text { such that } \\
\sin 2 \theta & =\frac{1}{\beta}=\frac{1}{\sqrt{1+\alpha^{2}}},
\end{aligned}
$$

up to local unitary transformations and Alice performs spin measurements along $Z$ and $X$, i.e., $A_{0}=|0\rangle\langle 0|-| 1\rangle\langle 1|$ and $A_{1}=|+\rangle\langle+|-|-\rangle\langle-|$ (or their local unitary equivalents), where $| \pm\rangle=\frac{|0\rangle \pm|1\rangle}{\sqrt{2}}$.

Proof. We are given that the joint state generates an assemblage that violates the tilted steering inequality maximally; therefore, the joint state is steerable and hence entangled. Using the arguments from the previous section, we can eliminate mixed entangled states, implying that the joint state is a pure entangled state. Also, we have shown that the maximal violation occurs for the state $|\psi(\theta)\rangle$ only if Alice and Bob make spin measurements along $Z$ and $X$, and for given values of $\beta$ and $\alpha$ satisfying $\beta^{2}=\alpha^{2}+1$ there is a unique $\theta$ in $(0, \pi / 4)$ given by $\sin 2 \theta=\frac{1}{\beta}$. Furthermore, any pure entangled state can be expressed as $|\psi(\theta)\rangle$ using Schmidt decomposition and for this form of the state the only measurements that achieve the maximal violation are the ones shown above; hence we can conclude that no other locally rotated state could achieve the maximal violation with the measurements defined above in the Schmidt bases.

Result 2. Maximal violation of the tilted steering inequality certifies any two-qubit pure entangled state; i.e., for the inequality $\alpha\left\langle A_{0}\right\rangle+\beta\left\langle A_{0} Z\right\rangle+\left\langle A_{1} X\right\rangle \leqslant \alpha+\sqrt{1+\beta^{2}}$ with $\beta^{2}=\alpha^{2}+1$, let the maximal violation be achieved by an assemblage generated by performing measurements on the joint pure state $|\psi\rangle_{A B} \in \mathcal{H}_{A} \otimes \mathcal{H}_{B}$ (where the dimension of the trusted side $B$ is 2 ) and measurement operators $\left\{M_{a \mid x}\right\}_{a, x}$ on Alice's side. Then there exists an isometry on Alice's side $\Phi: \mathcal{H}_{A} \rightarrow \mathcal{H}_{A} \otimes \mathcal{H}_{A^{\prime}}$, where the dimension of $\mathcal{H}_{A^{\prime}}$ is 2 , such that

$$
\begin{aligned}
\Phi\left(|\psi\rangle_{A B}\right) & =\mid \text { junk }\rangle_{A} \otimes|\psi(\theta)\rangle_{A^{\prime} B}, \\
\Phi\left(M_{a \mid x} \otimes I|\psi\rangle_{A B}\right) & =\mid \text { junk }\rangle_{A} \otimes\left(\tilde{M}_{a \mid x} \otimes I\right)|\psi(\theta)\rangle_{A^{\prime} B},
\end{aligned}
$$

where $|\psi(\theta)\rangle_{A^{\prime} B}$ is given by Eq. (A25) and measurement operators $\tilde{M}_{a \mid x}$ correspond to observables $A_{0}=\sigma_{z}$ and $A_{1}=\sigma_{x}$.

Proof. A similar result for state certification from the maximal violation of fine-grained steering inequality [49] was proved in [27]. According to Jordan's lemma (see [55] for proof), if there are operators $A_{0}$ and $A_{1}$ with eigenvalues \pm 1 acting on a Hilbert space $\mathcal{H}$ of dimension $d$, then there is a decomposition of $\mathcal{H}$ as a direct sum of subspaces $\mathcal{H}_{i}$ with dimension $d \leqslant 2$ each. That means $A_{0}$ and $A_{1}$ act within each subspace $\mathcal{H}_{i}$ and can be written as $A_{0}=\oplus_{i} A_{0}^{i}$ and $A_{1}=\oplus_{i} A_{1}^{i}$.

In our steering case, operators on the untrusted side (Alice) act on a Hilbert space $\mathcal{H}_{A}$ with dimension $d$. From Jordan's lemma, we can say that these observables act on subspace $\mathcal{H}_{A}^{i}$ with dimension $\leqslant 2$, i.e.

$$
\mathcal{H}_{A} \otimes \mathcal{H}_{B}=\oplus_{i}\left(\mathcal{H}_{A}^{i} \otimes \mathcal{H}_{B}\right) .
$$

Consider that $A_{x}=\Pi_{0 \mid A_{x}}-\Pi_{1 \mid A_{x}}$ with $x \in\{0,1\}$, where $\Pi_{a \mid A_{x}}$ is the projector acting on $\mathcal{H}_{A}$. Hence, we can write $\Pi_{a \mid A_{x}}=\oplus_{i} \Pi_{a \mid A_{x}}^{i}$ such that each $\Pi_{a \mid A_{x}}^{i}$ acts on $\mathcal{H}_{A}^{i}$ for all $a$ and 
$x$; we also denote as $\Pi^{i}=\Pi_{0 \mid A_{x}}^{i}+\Pi_{1 \mid A_{x}}^{i}$ the projector on $\mathcal{H}_{A}^{i}$. Also, we denote the projector associated with outcome $b$ of measurement $y$ as $\Pi_{b \mid B_{y}}$ acting on $\mathcal{H}_{B}$ of dimension 2 .

Now, for any state $\rho_{A B} \in \mathcal{B}\left(\mathcal{H}_{A} \otimes \mathcal{H}_{B}\right)$ we have

$$
\begin{aligned}
P(a, b \mid x, y) & =\operatorname{Tr}\left[\rho_{A B}\left(\Pi_{a \mid A_{x}} \otimes \Pi_{b \mid B_{y}}\right)\right] \\
& =\sum_{i} q_{i} \operatorname{Tr}\left[\rho_{A B}^{i}\left(\Pi_{a \mid A_{x}}^{i} \otimes \Pi_{b \mid B_{y}}\right)\right] \\
& =\sum_{i} q_{i} P_{i}(a, b \mid x, y),
\end{aligned}
$$

where $\quad q_{i}=\operatorname{Tr}\left[\rho_{A B}\left(\Pi^{i} \otimes I\right)\right] \geqslant 0 \forall i ; \sum_{i} q_{i}=1, \quad \rho_{A B}^{i}=$ $\frac{\left(\Pi^{i} \otimes I\right) \rho_{A B}\left(\Pi^{i} \otimes I\right)}{q_{i}} \in \mathcal{B}\left(\mathcal{H}_{A} \otimes \mathcal{H}_{B}\right)$ is, at most, a two-qubit state and $P_{i}(a, b \mid x, y)$ is the joint probability in the two-qubit subspace of Alice.

Using Eq. (A28), we can write the following correlation terms as

$$
\begin{aligned}
\left\langle A_{0} Z\right\rangle & =\sum_{i} q_{i}\left\langle A_{0} Z\right\rangle_{i}, \\
\left\langle A_{1} X\right\rangle & =\sum_{i} q_{i}\left\langle A_{1} X\right\rangle_{i}, \\
\left\langle A_{0}\right\rangle & =\sum_{i} q_{i}\left\langle A_{0}\right\rangle_{i},
\end{aligned}
$$

and as a consequence we can write the tilted steering expression as

$$
\begin{aligned}
I_{\alpha, \beta} & \equiv \alpha\left\langle A_{0}\right\rangle+\beta\left\langle A_{0} Z\right\rangle+\left\langle A_{1} X\right\rangle \\
& =\sum_{i} q_{i}\left(\alpha\left\langle A_{0}\right\rangle_{i}+\beta\left\langle A_{0} Z\right\rangle_{i}+\left\langle A_{1} X\right\rangle_{i}\right) \\
& =\sum_{i} q_{i} I_{\alpha, \beta}^{i} .
\end{aligned}
$$

Note that for the maximum value of $I_{\alpha, \beta}$ to be obtained it is necessary that, for all $i$ such that $q_{i} \neq 0$, the dimension of each $\mathcal{H}_{A}^{i}$ be equal to 2 . This implies that the effective dimension $d$ of the local Hilbert space of Alice $\mathcal{H}_{A}$ is even. Furthermore, from Eq. (A30) it follows that $I_{\alpha, \beta}$ is a convex sum over $I_{\alpha, \beta}^{i}$ and the maximal violation of $I_{\alpha, \beta}$ is obtained if and only if $I_{\alpha, \beta}^{i}$ is maximal $\left[=\sqrt{2\left(1+\beta^{2}+\alpha^{2}\right)}\right]$. From Lemma 1 , the maximal quantum value of $I_{\alpha, \beta}^{i}$ is achieved if and only if the shared two-qubit state $\rho_{A B}^{i}$ is a pure entangled state, i.e., $\rho_{A B}^{i}=$ $\left|\psi^{i}(\theta)\right\rangle\left\langle\psi^{i}(\theta)\right|$, where

$$
\left|\psi^{i}(\theta)\right\rangle=\cos \theta|2 i, 0\rangle+\sin \theta|2 i+1,1\rangle
$$

with $\sin 2 \theta=\frac{1}{\beta}=\frac{1}{\sqrt{1+\alpha^{2}}}$ and projectors given as $\Pi_{0 \mid A_{0}}^{i}=|2 i\rangle\left\langle 2 i\left|, \Pi_{1 \mid A_{0}}^{i}=\right| 2 i+1\right\rangle\left\langle 2 i+1\left|, \Pi_{0 \mid A_{1}}^{i}=\right|+{ }_{i}\right\rangle\left\langle+_{i}\right|$, and $\Pi_{1 \mid A_{1}}^{i}=\left|-{ }_{i}\right\rangle\left\langle-{ }_{i}|; \quad| \pm_{i}\right\rangle=\frac{|2 i\rangle \pm|2 i+1\rangle}{\sqrt{2}}$. Here, the state parameter $\theta$ is uniquely determined by $\beta$ (or $\alpha$ ) specified by the particular TSI being used for the certification task.

Hence, the state $|\psi\rangle_{A B} \in \mathcal{H}_{A} \otimes \mathcal{H}_{B}$ and measurement projection operators $\left\{M_{a \mid x}\right\}_{a, x}$ give the maximal violation if and only if they can be decomposed as

$$
|\psi\rangle_{A B}=\oplus_{i} \sqrt{q_{i}}\left|\psi^{i}(\theta)\right\rangle, \quad M_{a \mid x}=\oplus_{i} \Pi_{a \mid x}^{i},
$$

where $\sum_{i} q_{i}=1$ and $\left|\psi^{i}(\theta)\right\rangle$ is given by Eq. (A31).

Then one can append an ancilla qubit on Alice's side prepared in state $|0\rangle_{A^{\prime}}$ and define a local isometry as the map

$$
\begin{aligned}
\Phi\left|\oplus_{i} 2 i, 0\right\rangle_{A A^{\prime}} \longmapsto\left|\oplus_{i} 2 i, 0\right\rangle_{A A^{\prime}}, \\
\Phi\left|\oplus_{i} 2 i+1,0\right\rangle_{A A^{\prime}} \longmapsto\left|\oplus_{i} 2 i, 1\right\rangle_{A A^{\prime}},
\end{aligned}
$$

which can be used as

$$
\left.(\Phi \otimes I)|\psi\rangle_{A B}|0\rangle_{A^{\prime}}=\mid \text { junk }\right\rangle_{A} \otimes|\psi(\theta)\rangle_{A^{\prime} B}
$$

to extract the target state $|\psi(\theta)\rangle_{A^{\prime} B}$ [Eq. (A25)] from $|\psi\rangle_{A B}$.

\section{APPENDIX B: PROOF OF SDI CERTIFICATION FOR ARBITRARY BIPARTITE PURE ENTANGLED STATES}

Let us first describe the structure of the ideal assemblage that we would look for on Bob's side; then we will proceed to show how such a structure of the assemblage can be used for certifying the target state (6).

\section{Structure of the certifying assemblage}

In order to obtain the complete set of ideal assemblages that meet the sufficient condition for SDI certification, we need three measurement settings on Alice's side with $d$ outcomes each. The assemblage set generated from Alice's measurement settings $x \in\{0,1\}$ will be used to certify subspace projections of the form $c_{2 m}|2 m, 2 m\rangle+c_{2 m+1} \mid 2 m+1,2 m+$ $1\rangle$ and those generated from measurement settings $x \in\{0,2\}$ will certify projections of the form $c_{2 m+1}|2 m+1,2 m+1\rangle+$

\begin{tabular}{|c|c|c|c|c|c|}
\hline$\sigma_{2 m \mid 1}$ & $\langle 2 m|$ & $\langle 2 m+1|$ & $\sigma_{2 m+1 \mid 1}$ & $\langle 2 m|$ & $\langle 2 m+1|$ \\
\hline$|2 m\rangle$ & $\frac{c_{2 m}^{2}}{2}$ & $\frac{c_{2 m} c_{2 m+1}}{2}$ & $|2 m\rangle$ & $\frac{c_{2 m}^{2}}{2}$ & $-\frac{c_{2 m} c_{2 m+1}}{2}$ \\
\hline$|2 m+1\rangle$ & $\frac{c_{2 m} c_{2 m+1}}{2}$ & $\frac{c_{2 m+1}^{2}}{2}$ & $|2 m+1\rangle$ & $-\frac{c_{2 m} c_{2 m+1}}{2}$ & $\frac{c_{2 m+1}^{2}}{2}$ \\
\hline
\end{tabular}
$c_{2 m+2}|2 m+2,2 m+2\rangle$ of the target state.

Assemblage obtained from measurement $x=0$. For this case, we impose a condition that the assemblages corresponding to different outcomes only have diagonal nonzero terms:

$$
\sigma_{i \mid 0}=c_{i}^{2}|i\rangle\langle i| \quad \forall i \in\{0,1, \ldots, d-1\} .
$$

Assemblage obtained from measurement $x=1$. In this case, we require the assemblage to have certain nonzero nondiagonal terms along with diagonal terms. Specifically, the assemblages should have nonzero outer products between eigenvectors $|2 m\rangle$ and $|2 m+1\rangle$ in the computational basis (Tables I and II).

TABLE I. Nonzero elements of $\sigma_{2 m \mid 1}$ and $\sigma_{2 m+1 \mid 1} \forall m \in\left\{0, \ldots, \frac{d}{2}-1\right\}$ for even $d \geqslant 2$. 
TABLE II. Nonzero elements of $\sigma_{2 m \mid 1}$ and $\sigma_{2 m+1 \mid 1} \forall m \in\left\{0, \ldots, \frac{d-1}{2}-1\right\}$ for odd $d \geqslant 3$.

\begin{tabular}{|c|c|c|c|c|c|c|}
\hline$\sigma_{2 m \mid 1}$ & $\langle 2 m|$ & $\langle 2 m+1|$ & $\sigma_{2 m+1 \mid 1}$ & $\langle 2 m|$ & $\langle 2 m+1|$ & \\
\hline$|2 m\rangle$ & $\frac{c_{2 m}^{2}}{2}$ & $\frac{c_{2 m} c_{2 m+1}}{2}$ & $|2 m\rangle$ & $\frac{c_{2 m}^{2}}{2}$ & $-\frac{c_{2 m} c_{2 m+1}}{2}$ & $\sigma_{d-1 \mid 1}=c_{d-1}^{2}|d-1\rangle\langle d-1|$ \\
\hline$|2 m+1\rangle$ & $\frac{c_{2 m} c_{2 m+1}}{2}$ & $\frac{c_{2 m+1}^{2}}{2}$ & $|2 m+1\rangle$ & $-\frac{c_{2 m} c_{2 m+1}}{2}$ & $\frac{c_{2 m+1}^{2}}{2}$ & \\
\hline
\end{tabular}

Assemblage obtained from measurement $x=2$.. As in the previous case, this measurement will also give rise to an assemblage with nonzero nondiagonal elements but here they will be associated to the outer products between eigenvectors $|2 m+1 \bmod d\rangle$ and $|2 m+2 \bmod d\rangle$ in the computational basis (Tables III and IV).

\section{Ideal measurements on Alice's side}

We will now mention the ideal measurements on Alice's side that give rise to the required assemblages. For $x=0$, measurement is done in the computational basis, i.e., in the basis $\{|0\rangle,|1\rangle, \ldots,|d-1\rangle\}$; for $x=1$, for even $d$, measurement is done in the eigenbasis of observable $\oplus_{m=0}^{\frac{d}{2}-1}\left[\sigma_{x}\right]_{m}$, and for odd $d$, measurement is done in the eigenbasis of the observable $\oplus_{m=0}^{\frac{d-1}{2}-1}\left[\sigma_{x}\right]_{m} \oplus|d-1\rangle\langle d-1|$, where $\left[\sigma_{x}\right]_{m}=$ $|2 m\rangle\langle 2 m+1|+| 2 m+1\rangle\langle 2 m|$ is defined with respect to the basis $\{|2 m \bmod d\rangle,|2 m+1 \bmod d\rangle\}$; finally, for $x=2$, for even $d$, measurement is done in the eigenbasis of observable $\oplus_{m=0}^{\frac{d}{2}-1}\left[\sigma_{x}\right]_{m}^{\prime}$, and for odd $d$, measurement is done in the eigenbasis of the observable $|0\rangle\langle 0| \oplus_{m=0}^{\frac{d-1}{2}-1}\left[\sigma_{x}\right]_{m}^{\prime}$, where $\left[\sigma_{x}\right]_{m}^{\prime}=$ $|2 m+1\rangle\langle 2 m+2|+| 2 m+2\rangle\langle 2 m+1|$ is defined with respect to the basis $\{|2 m+1 \bmod d\rangle,|2 m+2 \bmod d\rangle\}$.

\section{Obtaining the sufficient conditions for SDI certification}

Analogous to the DI case $[6,17]$, we will show that the ideal assemblage structure can be used to obtain the condition given in Eq. (B 4) which is sufficient to show the existence of an isometry (Fig. 2 ) that certifies the target state. For this purpose we will construct projections $P_{A}^{(k)}$ and the unitaries $X_{A}^{(k)}$ using the measurement projection operators on Alice's side that give rise to the ideal assemblages. Let us denote Alice's projection operators for measurement $x$ and outcome $i$ by $\Pi_{i}^{A_{x}}$ and explore the consequences of imposing the ideal structure on the generated assemblage as shown in Appendix B 1.

Define the operator $A_{x, m}=\Pi_{2 m}^{A_{x}}-\Pi_{2 m+1}^{A_{x}}$ for $x \in\{0,1\}$; it can be seen that $\left(A_{x, m}\right)^{2}=\Pi_{2 m}^{A_{x}}+\Pi_{2 m+1}^{A_{x}}:=\mathbb{1}_{m}^{A_{x}}$. Further note that $\| \Pi_{i}^{A_{0}}|\psi\rangle \|=\sqrt{\left\langle\psi\left|\Pi_{i}^{A_{0}}\right| \psi\right\rangle}=\sqrt{\operatorname{tr}\left(\sigma_{i \mid A_{0}}\right)}=c_{i}$; similarly one can calculate $\| \mathbb{1}_{m}^{A_{x}}|\psi\rangle \|=\sqrt{c_{2 m}^{2}+c_{2 m+1}^{2}} \quad \forall x \in\{0,1\}$. Also, define Pauli $X, Z$, and identity operators on Bob's side for the basis $|2 m \bmod d\rangle,|2 m+1 \bmod d\rangle$ as $\quad Z_{B, m}=|2 m\rangle\langle 2 m|-| 2 m+1\rangle\left\langle 2 m+1\left|, \quad X_{B, m}=\right| 2 m\right\rangle$ $\langle 2 m+1|+| 2 m+1\rangle\langle 2 m|$, and $\mathbb{1}_{B, m}=|2 m\rangle\langle 2 m|+| 2 m+1\rangle$ $\langle 2 m+1|$.

Due to the structure imposed on the assemblages, we see that

$$
\begin{aligned}
I_{\alpha_{m}, \beta_{m}} & \equiv \alpha_{m}\left\langle\psi\left|A_{0, m} \mathbb{1}_{B, m}\right| \psi\right\rangle+\beta_{m}\left\langle\psi\left|A_{0, m} Z_{B, m}\right| \psi\right\rangle+\left\langle\psi\left|A_{1, m} X_{B, m}\right| \psi\right\rangle, \text { with } \beta_{m}^{2}=\alpha_{m}^{2}+1 \\
& =\alpha_{m} \operatorname{tr}\left(\sigma_{2 m \mid 0}-\sigma_{2 m+1 \mid 0}\right)+\beta_{m} \operatorname{tr}\left(\left(\sigma_{2 m \mid 0}-\sigma_{2 m+1 \mid 0}\right) Z_{B, m}\right)+\operatorname{tr}\left(\left(\sigma_{2 m \mid 1}-\sigma_{2 m+1 \mid 1}\right) X_{B, m}\right) \\
& =\alpha_{m}\left(c_{2 m}^{2}-c_{2 m+1}^{2}\right)+\beta_{m}\left(c_{2 m}^{2}+c_{2 m+1}^{2}\right)+2 c_{2 m} c_{2 m+1} \\
& =\left(\beta_{m}+\sqrt{1+\alpha_{m}^{2}}\right)\left(c_{2 m}^{2}+c_{2 m+1}^{2}\right) \\
& =2 \beta_{m}\left(c_{2 m}^{2}+c_{2 m+1}^{2}\right)\left[\text { note that } \sqrt{2\left(1+\alpha_{m}^{2}+\beta_{m}^{2}\right)}=2 \beta_{m}\right],
\end{aligned}
$$

where the state parameter $\theta_{m}$ is such that $\sin 2 \theta_{m}=\frac{1}{\beta_{m}}=\frac{2 c_{2 m} c_{2 m+1}}{c_{2 m}^{2}+c_{2 m+1}^{2}}$. This is not the maximal violation of the tilted steering inequality, but we can use a trick by defining the normalized state $\left|\psi_{m}\right\rangle=\frac{\mathbb{1}_{m}^{A_{0}}|\psi\rangle}{\sqrt{c_{2 m}^{2}+c_{2 m+1}^{2}}}$; consequently the assemblage modifies as $\sigma_{i \mid x, m}=\frac{\sigma_{i \mid x}}{c_{2 m}^{2}+c_{2 m+1}^{2}}$. As a result, the tilted steering expression evaluates to

$$
\begin{aligned}
I_{\alpha_{m}, \beta_{m}} & \equiv \alpha_{m}\left\langle\psi_{m}\left|A_{0, m} \mathbb{1}_{B, m}\right| \psi_{m}\right\rangle+\beta_{m}\left\langle\psi_{m}\left|A_{0, m} Z_{B, m}\right| \psi_{m}\right\rangle+\left\langle\psi_{m}\left|A_{1, m} X_{B, m}\right| \psi_{m}\right\rangle \\
& =\alpha_{m} \operatorname{tr}\left(\sigma_{2 m \mid 0, m}-\sigma_{2 m+1 \mid 0, m}\right)+\beta_{m} \operatorname{tr}\left(\left(\sigma_{2 m \mid 0, m}-\sigma_{2 m+1 \mid 0, m}\right) Z_{B, m}\right)+\operatorname{tr}\left(\left(\sigma_{2 m \mid 1, m}-\sigma_{2 m+1 \mid 1, m}\right) X_{B, m}\right) \\
& =\beta_{m}+\sqrt{1+\alpha_{m}^{2}}=2 \beta_{m},
\end{aligned}
$$

TABLE III. Nonzero elements of $\sigma_{2 m+1 \mid 2}$ and $\sigma_{2 m+2 \mid 2} \forall m \in\left\{0, \ldots, \frac{d}{2}-1\right\}$ for even $d \geqslant 2$.

\begin{tabular}{lcccc}
\hline \hline$\sigma_{2 m+1 \mid 2}$ & $\langle 2 m+1|$ & $\sigma_{2 m+2 \mid 2}$ & $\langle 2 m+1|$ & $\langle 2 m+2|$ \\
$|2 m+1\rangle$ & $\frac{c_{2 m+1}^{2}}{2}$ & $\frac{c_{2 m+1} c_{2 m+2}}{2}$ & $|2 m+1\rangle$ & $\frac{c_{2 m+1}^{2}}{2}$ \\
$|2 m+2\rangle$ & $\frac{c_{2 m+1} c_{2 m+2}}{2}$ & $\frac{c_{2 m+2}}{2}$ & $|2 m+2\rangle$ & $-\frac{c_{2 m+1} c_{2 m+2}}{2}$ \\
\hline \hline
\end{tabular}


TABLE IV. Nonzero elements of $\sigma_{2 m+1 \mid 2}$ and $\sigma_{2 m+2 \mid 2} \forall m \in\left\{0, \ldots, \frac{d-1}{2}-1\right\}$ for odd $d \geqslant 3$.

\begin{tabular}{|c|c|c|c|c|c|c|}
\hline \multirow{3}{*}{$\sigma_{0 \mid 2}=c_{0}^{2}|0\rangle\langle 0|$} & $\sigma_{2 m+1 \mid 2}$ & $\langle 2 m+1|$ & $\langle 2 m+2|$ & $\sigma_{2 m+2 \mid 2}$ & $\langle 2 m+1|$ & $\langle 2 m+2|$ \\
\hline & $|2 m+1\rangle$ & $\frac{c_{2 m+1}^{2}}{2}$ & $\frac{c_{2 m+1} c_{2 m+2}}{2}$ & $|2 m+1\rangle$ & $\frac{c_{2 m+1}^{2}}{2}$ & $-\frac{c_{2 m+1} c_{2 m+2}}{2}$ \\
\hline & $|2 m+2\rangle$ & $\frac{c_{2 m+1} c_{2 m+2}}{2}$ & $\frac{c_{2 m+2}^{2}}{2}$ & $|2 m+2\rangle$ & $-\frac{c_{2 m+1} c_{2 m+2}}{2}$ & $\frac{c_{2 m+2}^{2}}{2}$ \\
\hline
\end{tabular}

which is the maximal violation of the inequality given by $I_{\alpha_{m}}, \beta_{m}$. Then, by Lemma 1 , we can deduce that

$$
\begin{gathered}
\left|\psi_{m}\right\rangle=\frac{c_{2 m}|2 m, 2 m\rangle+c_{2 m+1}|2 m+1,2 m+1\rangle}{\sqrt{c_{2 m}^{2}+c_{2 m+1}^{2}}} \Rightarrow \mathbb{1}_{m}^{A_{0}}|\psi\rangle=c_{2 m}|2 m, 2 m\rangle+c_{2 m+1}|2 m+1,2 m+1\rangle, \\
A_{0, m}=|2 m\rangle\langle 2 m|-| 2 m+1\rangle\langle 2 m+1|, \\
A_{1, m}=|2 m\rangle\langle 2 m+1|+| 2 m+1\rangle\langle 2 m| .
\end{gathered}
$$

Now that we have the maximal violation, we need to define the unitarized versions of our operators as $A_{x, m}^{u}:=\mathbb{1}-\mathbb{1}_{m}^{A_{x}}+A_{x, m}$ and let $X_{A, m}^{u}:=A_{1, m}^{u}$; this unitarization is necessary to achieve the conditions of Lemma 2. As can be checked, the maximal violation in Eq. (B3) also holds with unitarized operators.

Next let us define the projections $P_{A}^{(2 m)}:=\left(\mathbb{1}_{m}^{A_{0}}+A_{0, m}\right) / 2=\Pi_{2 m}^{A_{0}}$ and $P_{A}^{(2 m+1)}:=\left(\mathbb{1}_{m}^{A_{0}}-A_{0, m}\right) / 2=\Pi_{2 m+1}^{A_{0}}$. So, for all $m$ and $k=2 m, 2 m+1$,

$$
\begin{aligned}
P_{A}^{(k)}|\psi\rangle & =\left(\mathbb{1}_{m}^{A_{0}}+(-1)^{k} A_{0, m}\right) / 2|\psi\rangle \\
& =\left(\mathbb{1}_{m}^{A_{0}}|\psi\rangle+(-1)^{k} A_{0, m}|\psi\rangle\right) / 2 \\
& =\left(\mathbb{1}_{m}^{A_{0}}|\psi\rangle+(-1)^{k} A_{0, m} \mathbb{1}_{m}^{A_{0}}|\psi\rangle\right) / 2 \quad\left(\text { since } A_{0, m}=A_{0, m} \mathbb{1}_{m}^{A_{0}}\right) \\
& =\left(c_{2 m}|2 m, 2 m\rangle+c_{2 m+1}|2 m+1,2 m+1\rangle+(-1)^{k}\left(c_{2 m}|2 m, 2 m\rangle-c_{2 m+1}|2 m+1,2 m+1\rangle\right)\right) / 2 \\
& =c_{k}|k, k\rangle .
\end{aligned}
$$

Furthermore, see that

$$
\begin{aligned}
X_{A, m}^{u} P_{A}^{(2 m+1)}|\psi\rangle & =X_{A, m}^{u} c_{2 m+1}|2 m+1,2 m+1\rangle \\
& =A_{1, m} c_{2 m+1}|2 m+1,2 m+1\rangle \\
& =c_{2 m+1}|2 m, 2 m+1\rangle .
\end{aligned}
$$

Similarly, we can perform analogous calculations for the operators: $A_{0, m}^{\prime}=\Pi_{2 m+1}^{A_{0}}-\Pi_{2 m+2}^{A_{0}}$ and $A_{1, m}^{\prime}=\Pi_{2 m+1}^{A_{2}}-\Pi_{2 m+2}^{A_{2}}$ for $x \in\{0,2\}$. Following the same procedure as shown above, we define the unitary operators $A_{x, m}^{\prime \prime}:=\mathbb{1}^{\prime}-\mathbb{1}_{m}^{A_{x}^{\prime}}+A_{x, m}^{\prime}$ and let $Y_{A, m}^{u}:=A_{1, m}^{\prime u}$; furthermore, we can obtain

$$
\begin{aligned}
P_{A}^{(k)}|\psi\rangle & =c_{k}|k, k\rangle \text { for all } k=2 m+1,2 m+2, \\
Y_{A, m}^{u} & =\mathbb{1}-\mathbb{1}_{m}^{A_{1}^{\prime}}+(|2 m+1\rangle\langle 2 m+2|+| 2 m+2\rangle\langle 2 m+1|), \\
Y_{A, m}^{u} P_{A}^{(2 m+2)}|\psi\rangle & =c_{2 m+2}|2 m+1,2 m+2\rangle .
\end{aligned}
$$

As the final step we construct the unitaries $X_{A}^{(k)}$ required in Lemma 2 from $X_{A, m}^{u}$ and $Y_{A, m}^{u}$ as

$$
X_{A}^{(k)}= \begin{cases}\mathbb{1} & \text { for } k=0 \\ X_{A, 0}^{u} Y_{A, 0}^{u} X_{A, 1}^{u} Y_{A, 1}^{u} \cdots X_{A, m-1}^{u} Y_{A, m-1}^{u} X_{A, m}^{u} & \text { for } k=2 m+1 \\ X_{A, 0}^{u} Y_{A, 0}^{u} X_{A, 1}^{u} Y_{A, 1}^{u} \cdots X_{A, m-1}^{u} Y_{A, m-1}^{u} & \text { for } k=2 m .\end{cases}
$$

Note that $X_{A}^{(k)}$ is unitary since it is composed of unitaries. Let us now verify the condition required in Lemma 2 , i.e.,

$$
X_{A}^{(k)} P_{A}^{(k)}|\psi\rangle=c_{k}|0, k\rangle
$$

for different cases.

For case $k=0$,

$$
\begin{aligned}
X_{A}^{(0)} P_{A}^{(0)}|\psi\rangle & =1 P_{A}^{(0)}|\psi\rangle \\
& =c_{0}|0,0\rangle .
\end{aligned}
$$

For case $k=2 m+1$,

$$
X_{A}^{(2 m+1)} P_{A}^{(2 m+1)}|\psi\rangle=X_{A, 0}^{u} Y_{A, 0}^{u} X_{A, 1}^{u} Y_{A, 1}^{u} \cdots X_{A, m-1}^{u} Y_{A, m-1}^{u} X_{A, m}^{u} P_{A}^{(2 m+1)}|\psi\rangle
$$




$$
\begin{aligned}
& =X_{A, 0}^{u} Y_{A, 0}^{u} X_{A, 1}^{u} Y_{A, 1}^{u} \cdots X_{A, m-1}^{u} Y_{A, m-1}^{u} c_{2 m+1}|2 m, 2 m+1\rangle \\
& =X_{A, 0}^{u} Y_{A, 0}^{u} X_{A, 1}^{u} Y_{A, 1}^{u} \cdots X_{A, m-1}^{u} c_{2 m+1}|2 m-1,2 m+1\rangle \\
& =X_{A, 0}^{u} Y_{A, 0}^{u} X_{A, 1}^{u} Y_{A, 1}^{u} \cdots c_{2 m+1}|2 m-2,2 m+1\rangle \\
& =\cdots \\
& =c_{2 m+1}|0,2 m+1\rangle .
\end{aligned}
$$

For the final case $k=2 m$,

$$
\begin{aligned}
X_{A}^{(2 m)} P_{A}^{(2 m)}|\psi\rangle & =X_{A, 0}^{u} Y_{A, 0}^{u} X_{A, 1}^{u} Y_{A, 1}^{u} \cdots X_{A, m-1}^{u} Y_{A, m-1}^{u} P_{A}^{(2 m)}|\psi\rangle \\
& =X_{A, 0}^{u} Y_{A, 0}^{u} X_{A, 1}^{u} Y_{A, 1}^{u} \cdots X_{A, m-1}^{u} Y_{A, m-1}^{u} c_{2 m}|2 m, 2 m\rangle \\
& =\cdots \\
& =c_{2 m}|0,2 m\rangle .
\end{aligned}
$$

\section{From the sufficient conditions to SDI state certification}

In the previous section we verified that condition (B9) holds. in the following lemma we claim that the condition is sufficient for showing the existence of an isometry on Alice's side, which can be used to extract out the target state (6) from the joint state between Alice and Bob.

Lemma 2. Suppose there exist unitary operators $X_{A}^{(k)}$ and projections $\left\{P_{A}^{(k)}\right\}_{k=0, \ldots, d-1}$ which form a complete orthogonal set and they satisfy the following condition:

$$
X_{A}^{(k)} P_{A}^{(k)}|\psi\rangle=c_{k}|0, k\rangle \quad \forall k
$$

Then there exists a local isometry on Alice's side $\Phi$ such that $\Phi(|\psi\rangle)=\mid$ junk $\rangle \otimes\left|\psi_{\text {target }}\right\rangle$, for some auxiliary state |junk $\rangle$.

Proof. We will consider a local isometry on Alice's side which is the $d$-dimensional generalization of SWAP isometry, introduced in [6]:

$$
\Phi:=R_{A A^{\prime}} \bar{F}_{A^{\prime}} S_{A A^{\prime}} F_{A^{\prime}},
$$

where $F$ is the quantum Fourier transform, $\bar{F}$ is the inverse quantum Fourier transform, $R_{A A^{\prime}}$ is defined as $R_{A A^{\prime}}|\psi\rangle_{A B}|k\rangle_{A^{\prime}}=X_{A}^{(k)}|\psi\rangle_{A B}|k\rangle_{A^{\prime}}$, and $S_{A A^{\prime}}$ is defined as $S_{A A^{\prime}}|\psi\rangle_{A B}|k\rangle_{A^{\prime}}=Z_{A}^{k}|\psi\rangle_{A B}|k\rangle_{A^{\prime}}$ where $Z_{A}:=\sum_{k=0}^{d-1} \omega^{k} P_{A}^{(k)}$. Let us now calculate the consequence of applying this isometry:

$$
\begin{aligned}
\Phi|\psi\rangle_{A B}|0\rangle_{A^{\prime}} & =R_{A A^{\prime}} \bar{F}_{A^{\prime}} S_{A A^{\prime}} F_{A^{\prime}}|\psi\rangle_{A B}|0\rangle_{A^{\prime}} \\
& =R_{A A^{\prime}} \bar{F}_{A^{\prime}} S_{A A^{\prime}} \frac{1}{\sqrt{d}} \sum_{k}|\psi\rangle_{A B}|k\rangle_{A^{\prime}}
\end{aligned}
$$

$$
\begin{aligned}
= & R_{A A^{\prime}} \bar{F}_{A^{\prime}} \frac{1}{\sqrt{d}} \sum_{k}\left(\sum_{j=0}^{d-1} \omega^{j} P_{A}^{(j)}\right)^{k}|\psi\rangle_{A B}|k\rangle_{A^{\prime}} \\
= & R_{A A^{\prime}} \bar{F}_{A^{\prime}} \frac{1}{\sqrt{d}} \sum_{k, j} \omega^{j k} P_{A}^{(j)}|\psi\rangle_{A B}|k\rangle_{A^{\prime}} \\
= & R_{A A^{\prime}} \frac{1}{d} \sum_{k, j, l} \omega^{j k} \omega^{-l k} P_{A}^{(j)}|\psi\rangle_{A B}|l\rangle_{A^{\prime}} \\
= & R_{A A^{\prime}} \sum_{j} P_{A}^{(j)}|\psi\rangle_{A B}|j\rangle_{A^{\prime}} \\
= & \sum_{j} X_{A}^{(j)} P_{A}^{(j)}|\psi\rangle_{A B}|j\rangle_{A^{\prime}} \\
= & \sum_{j} c_{j}|0, j\rangle_{A B}|j\rangle_{A^{\prime}} \\
& \times\left[\mathrm{B} 19{ }^{\prime} i n g\right. \text { the given condition (B13)] (B22) } \\
= & |0\rangle_{A} \otimes \sum_{j} c_{j}|j, j\rangle_{A^{\prime} B} \\
= & |j u n k\rangle_{A} \otimes\left|\psi_{\text {target }}\right\rangle_{A^{\prime} B} .
\end{aligned}
$$

Having certified the target state, the same isometry $\Phi$ [Eq. (B14)] can be used to certify Alice's ideal measurements. Using the same notations from previous sections, $A_{x, m}=\Pi_{2 m}^{A_{x}}-\Pi_{2 m+1}^{A_{x}}$ for $x \in\{0,1\}$ and we let the two-qubit ideal measurements be denoted by $\left[\sigma_{z}\right]_{m}$ for $x=0$ and $\left[\sigma_{x}\right]_{m}$ for $x=1$ on the $(2 m, 2 m+1)$ subspace (see Appendix B 2). Then, given that the observed assemblage has the ideal structure, consider the case $A_{x, m}$ for $x=1$ :

$$
\begin{aligned}
\Phi\left(A_{1, m}|\psi\rangle_{A B}|0\rangle_{A^{\prime}}\right) & =R_{A A^{\prime}} \sum_{j} P_{A}^{(j)} A_{1, m}|\psi\rangle_{A B}|j\rangle_{A^{\prime}} \quad \text { [continuing from (B20)] } \\
& =R_{A A^{\prime}} \sum_{j} P_{A}^{(j)} A_{1, m} \mathbb{1}_{m}^{A_{0}}|\psi\rangle_{A B}|j\rangle_{A^{\prime}} \\
& =R_{A A^{\prime}} \sum_{j} P_{A}^{(j)} A_{1, m}\left(c_{2 m}|2 m, 2 m\rangle_{A B}+c_{2 m+1}|2 m+1,2 m+1\rangle_{A B}\right)|j\rangle_{A^{\prime}}
\end{aligned}
$$




$$
\begin{aligned}
& =R_{A A^{\prime}} \sum_{j} P_{A}^{(j)}\left(c_{2 m}|2 m+1,2 m\rangle_{A B}+c_{2 m+1}|2 m, 2 m+1\rangle_{A B}\right)|j\rangle_{A^{\prime}} \quad \text { [from (B5)] } \\
& =R_{A A^{\prime}}\left(P_{A}^{(2 m+1)} c_{2 m}|2 m+1,2 m\rangle_{A B}|2 m+1\rangle_{A^{\prime}}+P_{A}^{(2 m)} c_{2 m+1}|2 m, 2 m+1\rangle_{A B}|2 m\rangle_{A^{\prime}}\right) \\
& =X_{A}^{(2 m+1)} P_{A}^{(2 m+1)} c_{2 m}|2 m+1,2 m\rangle_{A B}|2 m+1\rangle_{A^{\prime}}+X_{A}^{(2 m)} P_{A}^{(2 m)} c_{2 m+1}|2 m, 2 m+1\rangle_{A B}|2 m\rangle_{A^{\prime}} \\
& =c_{2 m}|0,2 m\rangle_{A B}|2 m+1\rangle_{A^{\prime}}+c_{2 m+1}|0,2 m+1\rangle_{A B}|2 m\rangle_{A^{\prime}} \\
& =|0\rangle_{A} \otimes\left(c_{2 m}|2 m+1,2 m\rangle_{A^{\prime} B}+c_{2 m+1}|2 m, 2 m+1\rangle_{A^{\prime} B}\right) \\
& =\mid \text { junk }\rangle_{A} \otimes\left[\sigma_{x}\right]_{m}\left|\psi_{\text {target }}\right\rangle_{A^{\prime} B} .
\end{aligned}
$$

Certification for subspace measurements $A_{x, m}$ can be shown similarly for the cases $x=0,2$.

\section{APPENDIX C: ROBUST SDI CERTIFICATION OF ALL PURE BIPARTITE MAXIMALLY ENTANGLED STATES}

We will adopt the assemblage-based robust SDI certification methodology introduced in [28] for proving our robust certification result for maximally entangled target states $\left|\bar{\psi}_{\text {target }}^{\max }\right\rangle:=\frac{1}{\sqrt{d}} \sum_{i=0}^{d-1}|i i\rangle$. First let us set up the reference experiment that will be studied in the rest of the section. Ideally we want the source to produce the reference state $\left|\bar{\psi}_{\text {target }}^{\max }\right\rangle=\frac{1}{\sqrt{d}} \sum_{i=0}^{d-1}|i i\rangle$ with $\bar{\rho}=\left|\bar{\psi}_{\text {target }}^{\max }\right\rangle\left\langle\bar{\psi}_{\text {target }}^{\max }\right|$ and Alice to perform the reference measurements as given in Appendix B 2 with reference projectors $\bar{M}_{a \mid x}$ for $a \in\{0, \ldots, d-$ $1\}$ and $x \in\{0,1,2\}$. The reference assemblages $\left\{\bar{\sigma}_{a \mid x}\right\}_{a, x}$ generated on Bob's side are as given in Appendix B 1 with $\bar{\sigma}_{a \mid x}=$ $\operatorname{tr}_{A}\left(\left(\bar{M}_{a \mid x} \otimes I\right) \bar{\rho}_{A B}\right)$.
The physical set of assemblages generated on Bob's side is denoted as $\sigma_{a \mid x}$ and the physical reduced state on Bob's side as $\rho_{B}$; furthermore, we consider the purification of the reduced state $\rho_{B}$ as $\left|\psi_{A B}\right\rangle$ for formulating and proving our robust certification results. Before proving our main result, let us describe three lemmas which will be useful in the proof.

Lemma 3 (borrowed from Lemma 2 of [28]). For any two vectors $|u\rangle,|v\rangle$ with $\||u\rangle \| \leqslant 1$ and $\||v\rangle \| \leqslant 1$, where $\||u\rangle \|=$ $\sqrt{\langle u \mid u\rangle}$, if $\||u\rangle-|v\rangle \| \leqslant \eta \leqslant 1$, then for another vector $|t\rangle$ with $\||t\rangle\|\leqslant \beta\|,(|u\rangle-|v\rangle)\langle t| \|_{1} \leqslant \beta \eta$ and $\||t\rangle(\langle u|-$ $\langle v|) \|_{1} \leqslant \beta \eta$.

Lemma 4. If $\left\|\sigma_{a \mid x}-\bar{\sigma}_{a \mid x}\right\|_{1} \leqslant \epsilon \forall a, x$ and $\mathrm{D}\left(\rho_{B}, \bar{\rho}_{B}\right) \leqslant \epsilon$ then

$$
\|\left(U_{A} M_{a \mid x} \otimes I_{B}\right)|\psi\rangle_{A B}-\left(U_{A} \otimes \bar{M}_{a \mid x}^{B}\right)|\psi\rangle_{A B} \| \leqslant 2 \sqrt{\epsilon} \quad \forall a, x,
$$

where $\bar{M}_{a \mid x}^{B}$ denotes the reference projector $\bar{M}_{a \mid x}$ acting on Bob's $d$-dimensional Hilbert space and $U_{A}$ is some unitary operator on Alice's side.

$$
\begin{aligned}
& \text { Proof. } \\
& \begin{aligned}
\|\left(U_{A} M_{a \mid x} \otimes I_{B}\right)|\psi\rangle_{A B}-\left(U_{A} \otimes \bar{M}_{a \mid x}^{B}\right)|\psi\rangle_{A B} \| & =\sqrt{\left\langle\psi_{A B}\left|M_{a \mid x} \otimes I_{B}\right| \psi\right\rangle_{A B}+\left\langle\psi_{A B}\left|I_{A} \otimes \bar{M}_{a \mid x}^{B}\right| \psi\right\rangle_{A B}-2\left\langle\psi_{A B}\left|M_{a \mid x} \otimes \bar{M}_{a \mid x}^{B}\right| \psi\right\rangle_{A B}} \\
& =\sqrt{\operatorname{Tr}_{B}\left(\sigma_{a \mid x}\right)+\operatorname{Tr}_{B}\left(\bar{M}_{a \mid x}^{B} \rho_{B}\right)-2 \operatorname{Tr}_{B}\left(\bar{M}_{a \mid x}^{B} \sigma_{a \mid x}\right)} \\
& \leqslant \sqrt{\frac{2}{d}+2 \epsilon-2 \operatorname{Tr}_{B}\left(\bar{M}_{a \mid x}^{B} \sigma_{a \mid x}\right)}
\end{aligned}
\end{aligned}
$$

follows from $\left|\operatorname{Tr}_{B}\left(\sigma_{a \mid x}-\bar{\sigma}_{a \mid x}\right)\right| \leqslant\left\|\sigma_{a \mid x}-\bar{\sigma}_{a \mid x}\right\|_{1} \leqslant \epsilon,\left|\operatorname{Tr}_{B}\left(\bar{M}_{a \mid x}^{B}\left(\rho_{B}-\bar{\rho}_{B}\right)\right)\right| \leqslant \mathrm{D}\left(\rho_{B}, \bar{\rho}_{B}\right) \leqslant \epsilon$ and since we are dealing with maximally entangled qudit states, $\operatorname{Tr}_{B}\left(\bar{\sigma}_{a \mid x}\right)=\operatorname{Tr}_{B}\left(\bar{M}_{a \mid x}^{B} \bar{\rho}_{B}\right)=\frac{1}{d} \quad \forall a, x$

$$
\leqslant \sqrt{\frac{2}{d}+2 \epsilon-2\left(\frac{1}{d}-\epsilon\right)}=2 \sqrt{\epsilon},
$$

since $\left|\operatorname{Tr}_{B}\left(\bar{M}_{a \mid x}^{B}\left(\sigma_{a \mid x}-\bar{\sigma}_{a \mid x}\right)\right)\right|=\left|\operatorname{Tr}_{B}\left(\bar{M}_{a \mid x}^{B}\left(\sigma_{a \mid x}\right)\right)-\frac{1}{d}\right| \leqslant\left\|\sigma_{a \mid x}-\bar{\sigma}_{a \mid x}\right\|_{1} \leqslant \epsilon \Rightarrow \operatorname{Tr}_{B}\left(\bar{M}_{a \mid x}^{B}\left(\sigma_{a \mid x}\right)\right) \geqslant \frac{1}{d}-\epsilon$.

Lemma 5. If $\left\|\sigma_{a \mid x}-\bar{\sigma}_{a \mid x}\right\|_{1} \leqslant \epsilon \forall a, x$ and $\mathrm{D}\left(\rho_{B}, \bar{\rho}_{B}\right) \leqslant \epsilon$ then

$$
\begin{gathered}
\|\left(U_{A} X_{A, m}^{u} \otimes I_{B}\right)|\psi\rangle_{A B}-\left(U_{A} \otimes \bar{X}_{B, m}^{u}\right)|\psi\rangle_{A B} \| \leqslant 4 \sqrt{\epsilon}, \\
\|\left(U_{A}^{\prime} Y_{A, m}^{u} \otimes I_{B}\right)|\psi\rangle_{A B}-\left(U_{A}^{\prime} \otimes \bar{Y}_{B, m}^{u}\right)|\psi\rangle_{A B} \| \leqslant 4 \sqrt{\epsilon},
\end{gathered}
$$

where $\bar{X}_{B, m}^{u}\left(\bar{Y}_{B, m}^{u}\right)$ denotes the reference unitary $\bar{X}_{A, m}^{u}\left(\bar{Y}_{A, m}^{u}\right)$ acting on Bob's $d$-dimensional Hilbert space and $U_{A}\left(U_{A}^{\prime}\right)$ is some unitary operator on Alice's side.

Proof. The proof follows closely that of Lemma 4 where instead of shifting the projector $M_{a \mid x}$, we shift the unitary $X_{A, m}^{u}\left(Y_{A, m}^{u}\right)$ to Bob's side. Recall from Appendix B 3 that we defined

$$
\begin{aligned}
& X_{A, m}^{u}:=A_{1, m}^{u}:=\mathbb{1}-\mathbb{1}_{m}^{A_{1}}+A_{1, m}=\mathbb{1}-2 \Pi_{2 m+1}^{A_{1}}, \\
& Y_{A, m}^{u}:=A_{2, m}^{u}:=\mathbb{1}-\mathbb{1}_{m}^{A_{2}}+A_{2, m}=\mathbb{1}-2 \Pi_{2 m+1}^{A_{2}},
\end{aligned}
$$


where $\Pi_{2 m+1}^{A_{1}}$ and $\Pi_{2 m+1}^{A_{2}}$ are the projectors $M_{a \mid x}$ for $a=2 m+1$ and $x=1,2$, respectively. Then we have

$$
\begin{aligned}
\|\left(U_{A} X_{A, m}^{u} \otimes I_{B}\right)|\psi\rangle_{A B}-\left(U_{A} \otimes \bar{X}_{B, m}^{u}\right)|\psi\rangle_{A B} \| & =\|\left(U_{A}\left(\mathbb{1}-2 M_{2 m+1 \mid 1}\right) \otimes I_{B}\right)|\psi\rangle_{A B}-\left(U_{A} \otimes\left(\mathbb{1}-2 \bar{M}_{2 m+1 \mid 1}^{B}\right)\right)|\psi\rangle_{A B} \| \\
& =2 \|\left(U_{A} \otimes \bar{M}_{2 m+1 \mid 1}^{B}\right)|\psi\rangle_{A B}-\left(U_{A} M_{2 m+1 \mid 1} \otimes I_{B}\right)|\psi\rangle_{A B} \| \\
& \leqslant 4 \sqrt{\epsilon}
\end{aligned}
$$

using Lemma 4. The proof for the case $Y_{A, m}^{u}$ proceeds similarly.

We will utilize these lemmas heavily in the proof of the main result in Sec. V, which proceeds as follows.

Proof. Recall that Bob has experimentally verified that

$$
\left\|\sigma_{a \mid x}-\bar{\sigma}_{a \mid x}\right\|_{1} \leqslant \epsilon \forall a, x \text { and } \mathrm{D}\left(\rho_{B}, \bar{\rho}_{B}\right) \leqslant \epsilon .
$$

Let us start by first bounding the distance between the states $|\Phi\rangle:=\Phi|\psi\rangle_{A B}|0\rangle_{A^{\prime}}$ and $\rho_{\text {junk }} \otimes \bar{\rho}_{A^{\prime} B}$ where $\rho_{\text {junk }}=\mid$ junk $\rangle\left\langle\right.$ junk $\left.\right|_{A}$ and $\bar{\rho}_{A^{\prime} B}=\left|\bar{\psi}_{\text {target }}^{\max }\right\rangle\left\langle\left.\bar{\psi}_{\text {target }}^{\max }\right|_{A^{\prime} B} \text {. As given in Eq. (B21), the effect of applying the local isometry } \Phi \text { on Alice's side is } \Phi \mid \psi\right\rangle_{A B}|0\rangle_{A^{\prime}}=$ $\sum_{j} X_{A}^{(j)} P_{A}^{(j)}|\psi\rangle_{A B}|j\rangle_{A^{\prime}}$ such that $P_{A}^{(j)}:=M_{j \mid 0}$ and $X_{A}^{(j)}$ are constructed as shown in Eq. (B8):

$$
X_{A}^{(j)}= \begin{cases}\mathbb{1} & \text { for } j=0 \\ X_{A, 0}^{u} Y_{A, 0}^{u} X_{A, 1}^{u} Y_{A, 1}^{u} \cdots X_{A, m-1}^{u} Y_{A, m-1}^{u} X_{A, m}^{u} & \text { for } j=2 m+1 \\ X_{A, 0}^{u} Y_{A, 0}^{u} X_{A, 1}^{u} Y_{A, 1}^{u} \cdots X_{A, m-1}^{u} Y_{A, m-1}^{u} & \text { for } j=2 m .\end{cases}
$$

Note that the physical unitaries $X_{A}^{(j)}$ and projectors $P_{A}^{(j)}$ do not satisfy condition (B13); however, the local isometry $\Phi$ can always be constructed such that Eq. (B21) is valid. Then,

$$
\begin{aligned}
\||\Phi\rangle\langle\Phi|-\rho_{\text {junk }} \otimes \bar{\rho}_{A^{\prime} B} \|_{1} & =\|\left(\sum_{j=0}^{d-1} X_{A}^{(j)} M_{j \mid 0}|\psi\rangle_{A B}|j\rangle_{A^{\prime}}\right)\langle\Phi|-\rho_{\text {junk }} \otimes \bar{\rho}_{A^{\prime} B} \|_{1} \\
& \leqslant 2 d \sqrt{\epsilon}+\|\left(\sum_{j=0}^{d-1} X_{A}^{(j)} \otimes \bar{M}_{j \mid 0}^{B}|\psi\rangle_{A B}|j\rangle_{A^{\prime}}\right)\langle\Phi|-\rho_{\text {junk }} \otimes \bar{\rho}_{A^{\prime} B} \|_{1},
\end{aligned}
$$

using Lemma 3 and Lemma 4 and the fact that $\||\Phi\rangle \|=1$. Now see that each term of the sum is of the form $X_{A}^{(j)} \otimes \bar{M}_{j \mid 0}^{B}|\psi\rangle_{A B}|j\rangle_{A^{\prime}}$ where $X_{A}^{(j)}$ is composed of $j$ unitaries $X_{A, m}^{u}$ which can be shifted to Bob's side using Lemma 5, such that

$$
\| X_{A}^{(j)} \otimes \bar{M}_{j \mid 0}^{B}|\psi\rangle_{A B}|j\rangle_{A^{\prime}}-I_{A} \otimes \bar{M}_{j \mid 0}^{B}\left(\bar{X}_{B}^{(j)}\right)^{\dagger}|\psi\rangle_{A B}|j\rangle_{A^{\prime}} \|_{1} \leqslant 4 j \sqrt{\epsilon}
$$

Continuing from Eq. (C5),

$$
\begin{aligned}
& 2 d \sqrt{\epsilon}+\|\left(\sum_{j=0}^{d-1} X_{A}^{(j)} \otimes \bar{M}_{j \mid 0}^{B}|\psi\rangle_{A B}|j\rangle_{A^{\prime}}\right)\langle\Phi|-\rho_{\text {junk }} \otimes \bar{\rho}_{A^{\prime} B} \|_{1} \\
& \leqslant 2 d \sqrt{\epsilon}+\sum_{j=0}^{d-1} 4 j \sqrt{\epsilon}+\|\left(\sum_{j=0}^{d-1} I_{A} \otimes \bar{M}_{j \mid 0}^{B}\left(\bar{X}_{B}^{(j)}\right)^{\dagger}|\psi\rangle_{A B}|j\rangle_{A^{\prime}}\right)\langle\Phi|-\rho_{\text {junk }} \otimes \bar{\rho}_{A^{\prime} B} \|_{1} \\
& =2 d^{2} \sqrt{\epsilon}+\|\left(\sum_{j=0}^{d-1} I_{A} \otimes \bar{M}_{j \mid 0}^{B}\left(\bar{X}_{B}^{(j)}\right)^{\dagger}|\psi\rangle_{A B}|j\rangle_{A^{\prime}}\right)\langle\Phi|-\rho_{\text {junk }} \otimes \bar{\rho}_{A^{\prime} B} \|_{1} .
\end{aligned}
$$

Now we apply the same strategy to shift the projectors $P_{A}^{(j)}$ and the unitary operators $X_{A}^{(j)}$ to Bob's side in $\langle\Phi|$, but before that we need $\| \sum_{j=0}^{d-1} I_{A} \otimes \bar{M}_{j \mid 0}^{B}\left(\bar{X}_{B}^{(j)}\right)^{\dagger}|\psi\rangle_{A B}|j\rangle_{A^{\prime}} \|$ :

$$
\begin{aligned}
\| \sum_{j=0}^{d-1} I_{A} \otimes \bar{M}_{j \mid 0}^{B}\left(\bar{X}_{B}^{(j)}\right)^{\dagger}|\psi\rangle_{A B}|j\rangle_{A^{\prime}} \| & =\sqrt{\sum_{j=0}^{d-1}\left\langle\psi_{A B}\left|I_{A} \otimes \bar{X}_{B}^{(j)} \bar{M}_{j \mid 0}^{B}\left(\bar{X}_{B}^{(j)}\right)^{\dagger}\right| \psi\right\rangle_{A B}} \\
& =\sqrt{\sum_{j=0}^{d-1}\left\langle\psi_{A B}\left|I_{A} \otimes \bar{M}_{0 \mid 0}^{B}\right| \psi\right\rangle_{A B}} \\
& =\sqrt{d\left(\operatorname{Tr}_{B}\left(\bar{M}_{0 \mid 0}^{B} \rho_{B}\right)\right)}
\end{aligned}
$$




$$
\begin{aligned}
& \leqslant \sqrt{d\left(\epsilon+\frac{1}{d}\right)} \\
& \leqslant 1+\frac{d \epsilon}{2} .
\end{aligned}
$$

Continuing from Eq. (C7),

$$
\begin{aligned}
& 2 d^{2} \sqrt{\epsilon}+\|\left(\sum_{j=0}^{d-1} I_{A} \otimes \bar{M}_{j \mid 0}^{B}\left(\bar{X}_{B}^{(j)}\right)^{\dagger}|\psi\rangle_{A B}|j\rangle_{A^{\prime}}\right)\langle\Phi|-\rho_{\text {junk }} \otimes \bar{\rho}_{A^{\prime} B} \|_{1} \\
& \leqslant 2 d^{2} \sqrt{\epsilon}+2 d^{2} \sqrt{\epsilon}\left(1+\frac{d \epsilon}{2}\right)+\|\left(\sum_{j=0}^{d-1} I_{A} \otimes \bar{M}_{j \mid 0}^{B}\left(\bar{X}_{B}^{(j)}\right)^{\dagger}|\psi\rangle_{A B}|j\rangle_{A^{\prime}}\right)\left(\sum _ { j = 0 } ^ { d - 1 } \left\langle\left.\psi\right|_{A B} I_{A} \otimes \bar{X}_{B}^{(j)} \bar{M}_{j \mid 0}^{B}\left\langle\left. j\right|_{A^{\prime}}\right)-\rho_{\text {junk }} \otimes \bar{\rho}_{A^{\prime} B}\|\|_{1}\right.\right. \\
& =4 d^{2} \sqrt{\epsilon}+d^{3} \epsilon \sqrt{\epsilon}+\|\left(\sum_{j=0}^{d-1}\left|j_{B}\right\rangle\left\langle 0_{B} \mid \psi\right\rangle_{A B}|j\rangle_{A^{\prime}}\right)\left(\sum_{j=0}^{d-1}\left\langle\psi_{A B} \mid 0_{B}\right\rangle\left\langle j_{B}\right|\left\langle\left. j\right|_{A^{\prime}}\right)-\rho_{\text {junk }} \otimes \bar{\rho}_{A^{\prime} B} \|_{1}\right. \\
& =4 d^{2} \sqrt{\epsilon}+d^{3} \epsilon \sqrt{\epsilon}+\|\left(\left\langle 0_{B} \mid \psi\right\rangle_{A B} \sqrt{d}\left|\bar{\psi}_{\text {target }}^{\max }\right\rangle_{A^{\prime} B}\right)\left(\left\langle\psi_{A B} \mid 0_{B}\right\rangle \sqrt{d}\left\langle\left.\bar{\psi}_{\text {target }}\right|_{A^{\prime} B}\right)-\rho_{\text {junk }} \otimes \bar{\rho}_{A^{\prime} B} \|_{1}\right. \\
& =4 d^{2} \sqrt{\epsilon}+d^{3} \epsilon \sqrt{\epsilon}+\left\|d\left\langle 0_{B} \mid \psi_{A B}\right\rangle\left\langle\psi_{A B} \mid 0_{B}\right\rangle \otimes \bar{\rho}_{A^{\prime} B}-\rho_{\text {junk }} \otimes \bar{\rho}_{A^{\prime} B}\right\|_{1} \\
& =4 d^{2} \sqrt{\epsilon}+d^{3} \epsilon \sqrt{\epsilon}+\left\|d\left\langle 0_{B} \mid \psi_{A B}\right\rangle\left\langle\psi_{A B} \mid 0_{B}\right\rangle-\rho_{\text {junk }}\right\|_{1} \\
& \leqslant 4 d^{2} \sqrt{\epsilon}+d^{3} \epsilon \sqrt{\epsilon}+d \epsilon
\end{aligned}
$$

where for obtaining the last inequality we assigned $\rho_{\text {junk }}=\left|\mathrm{junk}_{A}\right\rangle\left\langle\mathrm{junk}_{A}\right|$ to be the normalized state proportional to $\left\langle 0_{B} \mid \psi_{A B}\right\rangle$, i.e., $\left|j_{u n k_{A}}\right\rangle=\beta^{\frac{-1}{2}}\left\langle 0_{B} \mid \psi_{A B}\right\rangle$ with $\beta=\left\langle\psi_{A B} \mid 0_{B}\right\rangle\left\langle 0_{B} \mid \psi_{A B}\right\rangle$, and made the observation that $\left|\operatorname{Tr}_{B}\left(\left|0_{B}\right\rangle\left\langle 0_{B}\left|\rho_{B}-\right| 0_{B}\right\rangle\left\langle 0_{B}\right| \bar{\rho}_{B}\right)\right|=\mid \beta-$ $\frac{1}{d} \mid \leqslant \mathrm{D}\left(\rho_{B}, \bar{\rho}_{B}\right) \leqslant \epsilon$.

Having shown that $\mathrm{D}\left(|\Phi\rangle\langle\Phi|, \rho_{\text {junk }} \otimes \bar{\rho}_{A^{\prime} B}\right)=\frac{1}{2} \||\Phi\rangle\langle\Phi|-\rho_{\text {junk }} \otimes \bar{\rho}_{A^{\prime} B} \|_{1} \leqslant \frac{1}{2}\left(4 d^{2} \sqrt{\epsilon}+d^{3} \epsilon \sqrt{\epsilon}+d \epsilon\right)$, now we proceed to show the robustness result with measurements; i.e., we provide an upper bound for the distance

$$
\|\left|\Phi, M_{a \mid x}\right\rangle\left\langle\Phi, M_{a \mid x}\left|-\rho_{\text {junk }} \otimes\left(\bar{M}_{a \mid x} \otimes I_{B}\right)\right| \bar{\psi}_{\text {target }}^{\max }\right)\left\langle\bar{\psi}_{\text {target }}^{\max }\right|\left(\bar{M}_{a \mid x} \otimes I_{B}\right) \|_{1},
$$

where $\left|\Phi, M_{a \mid x}\right\rangle:=\Phi\left(M_{a \mid x} \otimes I_{B}\right)|\psi\rangle_{A B}|0\rangle_{A^{\prime}}$

Let us first consider the case for $x=0$, then $\left|\Phi, M_{a \mid 0}\right\rangle=\sum_{j} X_{A}^{(j)} P_{A}^{(j)}\left(M_{a \mid 0} \otimes I_{B}\right)|\psi\rangle_{A B}|j\rangle_{A^{\prime}}$ where $P_{A}^{(j)}=M_{j \mid 0}$ and for projectors we have $M_{j \mid 0} M_{a \mid 0}=\delta_{a, j} M_{a \mid 0}$. Then Eq. (C10) becomes

$$
\begin{aligned}
& \|\left|\Phi, M_{a \mid 0}\right\rangle\left\langle\Phi, M_{a \mid 0}\left|-\rho_{\text {junk }} \otimes\left(\bar{M}_{a \mid 0} \otimes I_{B}\right)\right| \bar{\psi}_{\text {target }}^{\text {max }}\right\rangle\left\langle\bar{\psi}_{\text {target }}^{\text {max }}\right|\left(\bar{M}_{a \mid 0} \otimes I_{B}\right) \|_{1} \\
& =\|\left(X_{A}^{(a)} P_{A}^{(a)}|\psi\rangle_{A B}|a\rangle_{A^{\prime}}\right)\left(\langle \psi | _ { A B } P _ { A } ^ { ( a ) } ( X _ { A } ^ { ( a ) } ) ^ { \dagger } \langle a | _ { A ^ { \prime } } ) - \rho _ { \text { junk } } \otimes \frac { 1 } { d } | a a \rangle \left\langle\left.a a\right|_{A^{\prime} B} \|_{1}\right.\right. \\
& \leqslant \\
& \quad 4 a \sqrt{\epsilon}+2 \sqrt{\epsilon}+(4 a \sqrt{\epsilon}+2 \sqrt{\epsilon}) \| I_{A} \otimes \bar{M}_{j \mid 0}^{B}\left(\bar{X}_{B}^{(j)}\right)^{\dagger}|\psi\rangle_{A B}|a\rangle_{A^{\prime}} \| \\
& \quad+\|\left(I_{A} \otimes \bar{M}_{a \mid 0}^{B}\left(\bar{X}_{B}^{(a)}\right)^{\dagger}|\psi\rangle_{A B}|a\rangle_{A^{\prime}}\right)\left(\langle \psi | _ { A B } I _ { A } \otimes \overline { X } _ { B } ^ { ( a ) } \overline { M } _ { a | 0 } ^ { B } \langle a | _ { A ^ { \prime } } ) - \rho _ { \text { junk } } \otimes \frac { 1 } { d } | a a \rangle \left\langle\left.a a\right|_{A^{\prime} B} \|_{1}\right.\right. \\
& \leqslant 2(4 a \sqrt{\epsilon}+2 \sqrt{\epsilon})+\left\|\left\langle 0_{B} \mid \psi_{A B}\right\rangle\left\langle\psi_{A B} \mid 0_{B}\right\rangle-\frac{\rho_{\text {junk }}}{d}\right\|_{1} \\
& \leqslant(8 a+4) \sqrt{\epsilon}+\epsilon \quad(\text { where } a \in\{0, \ldots, d-1\}) \\
& \leqslant(8 d-4) \sqrt{\epsilon}+\epsilon .
\end{aligned}
$$

Next consider the case for $x=1$ and $a=2 m$. The case for $a=2 m+1$ yields the same bound; then Eq. (C10) becomes

$$
\begin{aligned}
& \|\left|\Phi, M_{a \mid 1}\right\rangle\left\langle\Phi, M_{a \mid 1}\left|-\rho_{\text {junk }} \otimes\left(\bar{M}_{a \mid 1} \otimes I_{B}\right)\right| \bar{\psi}_{\text {target }}^{\max }\right\rangle\left\langle\bar{\psi}_{\text {target }}^{\max }\right|\left(\bar{M}_{a \mid 1} \otimes I_{B}\right) \|_{1} \\
& =\|\left(\sum_{j=0}^{d-1} X_{A}^{(j)} P_{A}^{(j)} M_{a \mid 1}|\psi\rangle_{A B}|j\rangle_{A^{\prime}}\right)\left\langle\Phi, M_{a \mid 1}\left|-\rho_{\text {junk }} \otimes \frac{1}{d}\right|+{ }_{2 m}+{ }_{2 m}\right\rangle\left\langle+_{2 m}+\left.{ }_{2 m}\right|_{A^{\prime} B} \|_{1}\right.
\end{aligned}
$$


where $\left|+{ }_{2 m}\right\rangle=\frac{|2 m\rangle+|2 m+1\rangle}{\sqrt{2}}$; repeatedly using Lemma 4 and Lemma 5 we get

$$
\begin{aligned}
& \leqslant \sum_{j=0}^{d-1}(2 \sqrt{\epsilon}+2 \sqrt{\epsilon}+4 j \sqrt{\epsilon})+\|\left(\sum_{j=0}^{d-1} \bar{M}_{a \mid 1}^{B} \bar{M}_{j \mid 0}^{B}\left(\bar{X}_{B}^{(j)}\right)^{\dagger}|\psi\rangle_{A B}|j\rangle_{A^{\prime}}\right)\left\langle\Phi, M_{a \mid 1}\left|-\rho_{\text {junk }} \otimes \frac{1}{d}\right|+2 m+{ }_{2 m}\right\rangle\left\langle+_{2 m}+\left.2 m\right|_{A^{\prime} B} \|_{1}\right. \\
& \leqslant 4\left(d^{2}+d\right) \sqrt{\epsilon}+\|\left(\left\langle 0_{B} \mid \psi_{A B}\right\rangle|+2 m+2 m\rangle_{A^{\prime} B}\right)\left(\left\langle\psi_{A B} \mid 0_{B}\right\rangle\langle+2 m+2 m|{ }_{A^{\prime} B}\right)-\rho_{\text {junk }} \otimes \frac{1}{d}|+2 m+2 m\rangle\left\langle+2 m+\left.2 m\right|_{A^{\prime} B} \|_{1}\right. \\
& =4\left(d^{2}+d\right) \sqrt{\epsilon}+\left\|\left\langle 0_{B} \mid \psi_{A B}\right\rangle\left\langle\psi_{A B} \mid 0_{B}\right\rangle-\frac{\rho_{\text {junk }}}{d}\right\|_{1} \\
& \leqslant 4\left(d^{2}+d\right) \sqrt{\epsilon}+\epsilon .
\end{aligned}
$$

Finally, the case for $x=2$ proceeds similarly, giving the same bound.

[1] J. Eisert, D. Hangleiter, N. Walk, I. Roth, D. Markham, R. Parekh, U. Chabaud, and E. Kashefi, Quantum certification and benchmarking, Nat. Rev. Phys. 2, 382 (2020).

[2] S. Popescu and D. Rohrlich, Generic quantum nonlocality, Phys. Lett. A 166, 293 (1992).

[3] D. Mayers and A. Yao, Self testing quantum apparatus, Quantum Inf. Comput. 4, 273 (2004).

[4] I. Šupić and J. Bowles, Self-testing of quantum systems: A review, Quantum 4, 337 (2020).

[5] T. H. Yang, T. Vértesi, J.-D. Bancal, V. Scarani, and M. Navascués, Robust and Versatile Black-Box Certification of Quantum Devices, Phys. Rev. Lett. 113, 040401 (2014).

[6] T. H. Yang and M. Navascués, Robust self-testing of unknown quantum systems into any entangled two-qubit states, Phys. Rev. A 87, 050102(R) (2013).

[7] C. Bamps and S. Pironio, Sum-of-squares decompositions for a family of Clauser-Horne-Shimony-Holt-like inequalities and their application to self-testing, Phys. Rev. A 91, 052111 (2015).

[8] P. Sekatski, J.-D. Bancal, S. Wagner, and N. Sangouard, Certifying the Building Blocks of Quantum Computers from Bell's Theorem, Phys. Rev. Lett. 121, 180505 (2018).

[9] S. Pironio, A. Acín, S. Massar, A. B. de La Giroday, D. N. Matsukevich, P. Maunz, S. Olmschenk, D. Hayes, L. Luo, T. A. Manning et al., Random numbers certified by Bell's theorem, Nature (London) 464, 1021 (2010).

[10] O. Andersson, P. Badziag, I. Dumitru, and A. Cabello, Deviceindependent certification of two bits of randomness from one entangled bit and Gisin's elegant bell inequality, Phys. Rev. A 97, 012314 (2018).

[11] S. Gómez, A. Mattar, I. Machuca, E. S. Gómez, D. Cavalcanti, O. J. Farías, A. Acín, and G. Lima, Experimental investigation of partially entangled states for device-independent randomness generation and self-testing protocols, Phys. Rev. A 99, 032108 (2019).

[12] D. Mayers and A. Yao, Quantum cryptography with imperfect apparatus, in Proceedings 39th Annual Symposium on Foundations of Computer Science (IEEE, 1998), pp. 503-509.

[13] R. Jain, C. A. Miller, and Y. Shi, Parallel device-independent quantum key distribution, IEEE Trans. Inf. Theory 66, 5567 (2020).
[14] J. Bowles, I. Šupić, D. Cavalcanti, and A. Acín, DeviceIndependent Entanglement Certification of All Entangled States, Phys. Rev. Lett. 121, 180503 (2018).

[15] J. Bowles, I. Šupić, D. Cavalcanti, and A. Acín, Self-testing of Pauli observables for device-independent entanglement certification, Phys. Rev. A 98, 042336 (2018).

[16] N. Brunner, D. Cavalcanti, S. Pironio, V. Scarani, and S. Wehner, Bell nonlocality, Rev. Mod. Phys. 86, 419 (2014).

[17] A. Coladangelo, K. T. Goh, and V. Scarani, All pure bipartite entangled states can be self-tested, Nat. Commun. 8, 15485 (2017).

[18] I. Šupić, A. Coladangelo, R. Augusiak, and A. Acín, Selftesting multipartite entangled states through projections onto two systems, New J. Phys. 20, 083041 (2018).

[19] B. W. Reichardt, F. Unger, and U. Vazirani, Classical command of quantum systems, Nature (London) 496, 456 (2013).

[20] M. McKague, Self-testing in parallel, New J. Phys. 18, 045013 (2016).

[21] A. Coladangelo, Parallel self-testing of (tilted) EPR pairs via copies of (tilted) $\mathrm{CHSH}$ and the magic square game, Quantum Inf. Comput. 17, 831 (2017).

[22] S. Breiner, A. Kalev, and C. A. Miller, Parallel self-testing of the GHZ state with a proof by diagrams, EPTCS 287, 43 (2019).

[23] K. Bharti, M. Ray, A. Varvitsiotis, N. A. Warsi, A. Cabello, and L.-C. Kwek, Robust Self-Testing of Quantum Systems via Noncontextuality Inequalities, Phys. Rev. Lett. 122, 250403 (2019).

[24] K. Bharti, M. Ray, A. Varvitsiotis, A. Cabello, and L.-C. Kwek, Local certification of programmable quantum devices of arbitrary high dimensionality, arXiv:1911.09448.

[25] A. Tavakoli, J. Kaniewski, T. Vértesi, D. Rosset, and N. Brunner, Self-testing quantum states and measurements in the prepare-and-measure scenario, Phys. Rev. A 98, 062307 (2018).

[26] F. Magniez, D. Mayers, M. Mosca, and H. Ollivier, Self-testing of quantum circuits, in International Colloquium on Automata, Languages, and Programming (Springer, Berlin, 2006), pp. 72-83.

[27] S. Goswami, B. Bhattacharya, D. Das, S. Sasmal, C. Jebaratnam, and A. S. Majumdar, One-sided deviceindependent self-testing of any pure two-qubit entangled state, Phys. Rev. A 98, 022311(R) (2018). 
[28] I. Šupić and M. J. Hoban, Self-testing through EPR-steering, New J. Phys. 18, 075006 (2016).

[29] A. Gheorghiu, P. Wallden, and E. Kashefi, Rigidity of quantum steering and one-sided device-independent verifiable quantum computation, New J. Phys. 19, 023043 (2017).

[30] A. Einstein, B. Podolsky, and N. Rosen, Can quantummechanical description of physical reality be considered complete? Phys. Rev. 47, 777 (1935).

[31] E. Schrödinger, Discussion of probability relations between separated systems, Math. Proc. Cambridge Philos. Soc. 31, 555 (1935).

[32] E. Schrödinger, Probability relations between separated systems, Math. Proc. Cambridge Philos. Soc. 32, 446 (1936).

[33] C. Branciard, E. G. Cavalcanti, S. P. Walborn, V. Scarani, and H. M. Wiseman, One-sided device-independent quantum key distribution: Security, feasibility, and the connection with steering, Phys. Rev. A 85, 010301(R) (2012).

[34] C. H. Bennett, G. Brassard, and N. D. Mermin, Quantum Cryptography without Bell's Theorem, Phys. Rev. Lett. 68, 557 (1992).

[35] Y. Z. Law, L. P. Thinh, J.-D. Bancal, and V. Scarani, Quantum randomness extraction for various levels of characterization of the devices, J. Phys. A: Math. Theor. 47, 424028 (2014).

[36] E. Passaro, D. Cavalcanti, P. Skrzypczyk, and A. Acín, Optimal randomness certification in the quantum steering and prepareand-measure scenarios, New J. Phys. 17, 113010 (2015).

[37] P. Skrzypczyk and D. Cavalcanti, Maximal Randomness Generation from Steering Inequality Violations Using Qudits, Phys. Rev. Lett. 120, 260401 (2018).

[38] R. Uola, A. C. S. Costa, H. C. Nguyen, and O. Gühne, Quantum steering, Rev. Mod. Phys. 92, 015001 (2020).

[39] D. Cavalcanti and P. Skrzypczyk, Quantum steering: A review with focus on semidefinite programming, Rep. Prog. Phys. 80, 024001 (2016).

[40] J. F. Clauser, M. A. Horne, A. Shimony, and R. A. Holt, Proposed Experiment to Test Local Hidden-Variable Theories, Phys. Rev. Lett. 23, 880 (1969).

[41] E. G. Cavalcanti, S. J. Jones, H. M. Wiseman, and M. D. Reid, Experimental criteria for steering and the Einstein-PodolskyRosen paradox, Phys. Rev. A 80, 032112 (2009).

[42] Z. Bian, A. S. Majumdar, C. Jebarathinam, K. Wang, L. Xiao, X. Zhan, Y. Zhang, and P. Xue, Experimental demonstration of one-sided device-independent self-testing of any pure two-qubit entangled state, Phys. Rev. A 101, 020301(R) (2020).

[43] V. Händchen, T. Eberle, S. Steinlechner, A. Samblowski, T. Franz, R. F. Werner, and R. Schnabel, Observation of oneway Einstein-Podolsky-Rosen steering, Nat. Photonics 6, 596 (2012).

[44] D. J. Saunders, S. J. Jones, H. M. Wiseman, and G. J. Pryde, Experimental EPR-steering using Bell-local states, Nat. Phys. 6, 845 (2010).

[45] M. Malik, V. Srivastav, N. H. Valencia, W. McCutcheon, S. Designolle, R. K. Uola, and N. Brunner, Genuine highdimensional quantum steering, in Optical and Quantum Sensing and Precision Metrology, Vol. 11700 (International Society for Optics and Photonics, Bellingham, WA, 2021), p. 117001H.

[46] L. Mančinska, J. Prakash, and C. Schafhauser, Constant-sized robust self-tests for states and measurements of unbounded dimension, arXiv:2103.01729.

[47] H. Fu, Constant-sized correlations are sufficient to robustly selftest maximally entangled states with unbounded dimension, arXiv:1911.01494.

[48] A. Gheorghiu, E. Kashefi, and P. Wallden, Robustness and device independence of verifiable blind quantum computing, New J. Phys. 17, 083040 (2015).

[49] T. Pramanik, M. Kaplan, and A. S. Majumdar, Fine-grained Einstein-Podolsky-Rosen-steering inequalities, Phys. Rev. A 90, 050305(R) (2014).

[50] E. G. Cavalcanti, C. J. Foster, M. Fuwa, and H. M. Wiseman, Analog of the Clauser-Horne-Shimony-Holt inequality for steering, J, Opt. Soc. Am. B 32, A74 (2015).

[51] H. M. Wiseman, S. J. Jones, and A. C. Doherty, Steering, Entanglement, Nonlocality, and the Einstein-Podolsky-Rosen Paradox, Phys. Rev. Lett. 98, 140402 (2007).

[52] P. Skrzypczyk, M. Navascués, and D. Cavalcanti, Quantifying Einstein-Podolsky-Rosen Steering, Phys. Rev. Lett. 112, 180404 (2014).

[53] R. Gallego and L. Aolita, Resource Theory of Steering, Phys. Rev. X 5, 041008 (2015).

[54] A. Acín, S. Massar, and S. Pironio, Randomness Versus Nonlocality and Entanglement, Phys. Rev. Lett. 108, 100402 (2012).

[55] L. Masanes, Asymptotic Violation of Bell Inequalities and Distillability, Phys. Rev. Lett. 97, 050503 (2006). 